\title{
A novel phylogenetic analysis and machine learning predict pathogenicity of human mtDNA variants
}

Bala Anı Akpınar ${ }^{1 \dagger}$, Paul O. Carlson, Ville O. Paavilainen ${ }^{1}$, and Cory D. Dunn ${ }^{1 \dagger}$

${ }^{1}$ Institute of Biotechnology, Helsinki Institute of Life Science, University of Helsinki, Helsinki, 00014, Finland

${ }^{\dagger}$ Corresponding authors

Correspondence:

Bala Anı Akpınar, Ph.D.

P.O. Box 56

University of Helsinki

00014 Finland

Email: ani.akpinar@helsinki.fi

Phone: +358 503119307

or

Cory Dunn, Ph.D.

P.O. Box 56

University of Helsinki

00014 Finland

Email: cory.dunn@helsinki.fi

Phone: +358 503119307 


\section{ABSTRACT}

Linking mitochondrial DNA (mtDNA) variation to clinical outcomes remains a formidable challenge. Diagnosis of mitochondrial disease is hampered by the multicopy nature and potential heteroplasmy of the mitochondrial genome, differential distribution of mutant mtDNAs among various tissues, genetic interactions among alleles, and environmental effects. Here, we describe a new approach to the assessment of which mtDNA variants may be pathogenic. Our method takes advantage of site-specific conservation and variant acceptability metrics that minimize previous classification limitations. Using our novel features, we deploy machine learning to predict the pathogenicity of thousands of human mtDNA variants. Our work demonstrates that a substantial fraction of mtDNA changes not yet characterized as harmful are, in fact, likely to be deleterious. Our findings will be of direct relevance to those at risk of mitochondria-associated metabolic disease. 


\section{INTRODUCTION}

Because of the critical roles that mitochondria play in metabolism and bioenergetics, mutation of mitochondria-localized proteins and ribonucleic acids can adversely affect human health (Alston et al, 2017; Suomalainen \& Battersby, 2018; Khan et al, 2020; Russell et al, 2020). Indeed, at least one in 5000 people (Gorman et al, 2015) is estimated to be overtly affected by mitochondrial disease. While a very limited number of mitochondrial DNA (mtDNA) lesions can be directly linked to human illness, the clinical outcome for many other mtDNA changes remains ambiguous (Vento \& Pappa, 2013). Heteroplasmy among the hundreds of mitochondrial DNA (mtDNA) molecules found within a cell (Stewart \& Chinnery, 2015; Hahn \& Zuryn, 2019; Wei \& Chinnery, 2020), differential distribution of disease-causing mtDNA among tissues (Boulet et al, 1992), and modifier alleles within the mitochondrial genome (Wei et al, 2017; Elliott et al, 2008) magnify the difficulty of interpreting different mtDNA alterations. Mito-nuclear interactions and environmental effects may also determine the outcome of mitochondrial DNA mutations (Wolff et al, 2014; Hill et al, 2019; Matilainen et al, 2017; Turnbull et al, 2018). Beyond the obvious importance of resolving the genetic etiology of symptoms presented in a clinical setting, the rapidly increasing prominence of direct-to-consumer genetic testing (Phillips et al, 2018) calls for an improved understanding of which mtDNA polymorphisms might affect human health (Blell \& Hunter, 2019).

Simple tabulation of mtDNA variants found among healthy or sick individuals (Whiffin et al, 2017) may be of limited utility in predicting how harmful a variant may be. Differing, strand-specific mutational propensities for mtDNA nucleotides at different locations within the molecule (Tanaka \& Ozawa, 1994; Faith \& Pollock, 2003; Reyes et al, 1998) should be taken into account when assessing population-wide data, yet allele frequencies are rarely, if ever, normalized in this way. Population sampling biases and recent population bottleneck effects can lead to misinterpretation of variant frequencies (Zuk et al, 2014; Chheda et al, 2017; Keinan \& Clark, 2012; Landry et al, 2018; Pirastu et al, 2020). Mildly deleterious variants arising in a population are slow to be removed by selection (Nachman, 1998; Nachman et al, 1996), leading to a false prediction of variant benignancy. Finally, a lack of selection against variants that might act in a deleterious manner at the post-reproductive stage of life also makes likely the possibility that some mtDNA changes will contribute to age-related phenotypes while avoiding overt association with mitochondrial disease (Maklakov et al, 2015; Medawar, 1952; Cui et al, 2019; Williams, 1957; Wallace, 1994).

Examining evolutionary conservation by use of multiple sequence alignments offers important assistance when predicting a variant's potential pathogenicity (Raychaudhuri, 2011; Tang \& Thomas, 2016a). However, caveats are also associated with predicting mutation outcome by the use of these alignments. First, while knowledge of amino acid physico-chemical properties is widely considered to be informative regarding whether an amino acid substitution may or may not have a damaging effect on protein function (Dayhoff 
et al, 1978), the site-specific acceptability of a given substitution is ultimately decided within the context of its local protein environment (Zuckerkandl \& Pauling, 1965). Second, sampling biases and improper clade selection may lead to inaccurate clinical interpretations regarding the relative acceptability of specific variants (Zuk et al, 2014; Chheda et al, 2017; Keinan \& Clark, 2012; Landry et al, 2018). Third, alignment (Kawrykow et al, 2012; lantorno et al, 2014) and sequencing errors (Chen et al, 2017; Smith, 2019) may falsely indicate the acceptability of a particular mtDNA substitution.

Here, we have deployed a methodology to calculate, by a novel analysis of available mammalian genomes, the relative conservation of human mtDNA-encoded positions. Moreover, we infer ancestral direct substitutions within mammals and test whether they match substitutions from the human reference sequence, providing further knowledge regarding the potential pathogenicity of any human mtDNA substitution. By subsequent application of machine learning, we demonstrate that a surprising number of uncharacterized mtDNA mutations carried by humans are likely to promote disease. We provide our predictions, which should be of great utility to clinicians and to those studying mitochondrial disease.

\section{RESULTS}

\section{Mapping apparent substitutions to a phylogenetic tree allows calculation of relative positional conservation in mtDNA-encoded proteins and RNAs}

We previously developed an empirical method for detection and quantification of mtDNA substitutions mapped to the edges of a phylogenetic tree (Dunn et al, 2020). Here, we have extended our approach toward prediction of human mitochondrial variant pathogenicity. First, we retrieved full mammalian mtDNA sequences from the National Center for Biotechnology Information Reference Sequence (NCBI RefSeq) database and extracted each RNA or protein-coding gene using the Homo sapiens reference mtDNA as a guide. Next, we aligned the resulting protein, tRNA, and rRNA sequences, concatenated the sequences of each species based upon molecule class, and generated phylogenetic trees using a maximum likelihood approach. Following tree generation, we performed ancestral prediction to reconstruct the character values of each position at every bifurcating node. Using the sequences of extant species and the predicted ancestral node values, we subsequently analyzed each edge of the tree for the presence or absence of substitutions at each aligned human position. We subsequently sum all substitutions at a given position that occur along all tree edges to generate a new metric, the total substitution score (TSS, Figure 1A). The TSS should surpass metrics that consider positional character frequencies derived from multiple sequence alignments as a proxy of conservation, as character frequencies are highly sensitive to sampling biases among input sequences. Moreover, many site-specific measurements of variability, such as Shannon entropy, are limited in dynamic range and 
benefit minimally from the rapid increase in available genomic information. In contrast, the dynamic range of the TSS is very wide, and potentially unlimited, continuously benefitting from the accretion of new sequence information.

Furthermore, by excluding edges from analysis that lead directly to extant sequences, one can further minimize effects of alignment errors and sequencing errors that may lead to eventual misinterpretation of variant pathogenicity. Moreover, mutations mapped to internal edges are more likely to represent fixed changes informative for the purposes of disease prediction, while polymorphisms that have not yet been subject to selection of sufficient strength or duration might be expected to complicate predictions of variant pathogenicity (Nachman et al, 1996; Nachman, 1998). Summation of substitutions only at these internal edges provides an internal substitution score (ISS, Figure 1B).

When calculated for protein and RNA sites encoded by mammalian mtDNA, it is clear that the TSS (and the ISS, not shown) provides an excellent readout of relative conservation at, and consequent functional importance of, each alignment position. When comparing TSS data from different mtDNA-encoded proteins, our findings are consistent with previous results, obtained by alternative methodologies, demonstrating that the core, mtDNAencoded subunits of Complexes III and IV tend to be the most conserved, while positions within the mtDNA-encoded polypeptides of Complex I and Complex $\mathrm{V}$ tend to be less well conserved (da Fonseca et al, 2008; Nabholz et al, 2013) (Figure 2A). Examination of the structures of these complexes indicate that, indeed, the most conserved residues are preferentially localized near the key catalytic regions of each complex (not shown). Within each protein, there was, as expected, a spectrum of site conservation values, also illustrated by plotting a distribution of TSS values across each polypeptide (Figure S1). Nearly all analyzed protein positions appeared to be under some selective pressure and are not saturated with mutations, with TSS values existing far from the maximal values that can be achieved within this phylogenetic analysis of mammals. Selective pressure on most aligned sites is also observed when examining mtDNA-encoded tRNAs and rRNAs (Figure 2B and Figure S2).

Beyond summation of substitutions across a phylogenetic tree, the inferred ancestral and descendent characters at each edge of the phylogenetic tree can also be examined following generation of the substitution map and can provide important information regarding what changes to mtDNA-encoded macromolecules might be deleterious or not. Specifically, if an inferred direct substitution from the human reference character to the mutant character (or the inverse, assuming the time-reversibility of character substitutions) is predicted along the edge of a phylogenetic tree, then such a change at a given position might be expected to be less deleterious than an inferred direct substitution to or from the human character that was never encountered over the evolutionary history of a clade. In contrast, the simple presence or absence of a character at an alignment position, without 
the context of its ancestral character, will fail to reflect epistatic relationships that involve the position of interest (Kimura, 1985; Kondrashov et al, 2002; Marini et al, 2010). A further focus upon inferred direct substitutions occurring at internal tree edges, which we call internal inferred direct substitutions (IIDSs) (Figure 1C), avoids the confounding effects of sequencing and alignment errors (Chen et al, 2017; Smith, 2019) and of the abovementioned, incomplete selection against newly arisen, mildly deleterious alleles (Nachman et al, 1996; Nachman, 1998).

\section{Substitution scores and inferred direct substitutions can be linked to human mtDNA variant pathogenicity}

Since summation of detected substitutions across a phylogenetic tree provides a robust measure of relative conservation at different macromolecular positions, we were confident that a phylogenetic analysis that includes TSSs would also provide information about the pathogenicity of human mtDNA variants. To test this possibility, we focused our attention upon harmful substitutions annotated within the MITOMAP database of pathogenic mtDNA alterations (Lott et al, 2013). Indeed, we detected a clear relationship between confirmed pathogenicity and conservation at mtDNA-encoded amino acid positions, as reflected by positional TSSs (Figure 2C). Similarly, there was a strong link between tRNA mutation pathogenicity and TSSs obtained from mammalian tRNA data (Figure 2D). The paucity of confirmed pathogenic mitochondrial rRNA mutations in the MITOMAP database made comparisons using this class of molecules impractical, yet we expect future confirmation of additional pathogenic mutations in mitochondria-encoded rRNAs to permit further analyses. Together, findings obtained by phylogenetic analysis of mitochondriaencoded proteins and tRNAs indicate that TSSs are a valuable asset when predicting which mtDNA mutations might lead to disease.

To test the possibility that IIDSs, like TSSs, may predict pathogenicity, we compared human variants confirmed to cause disease to those variants currently labelled as polymorphisms within the MITOMAP database. Pathogenic human amino acid changes from the reference sequence are rarely encountered among the internal edges of a mammalian phylogenetic tree (Figure 3A). In contrast, reportedly polymorphic alleles were much more likely to have the amino acid substitution identifiable along internal edges of the mammalian tree. For nucleotide substitutions in tRNAs, a similar analysis comparing confirmed pathogenic variants and variants annotated as polymorphisms detected no statistically significant relationship between pathogenicity and the detection of IIDSs.

\section{Widespread, uncharacterized pathogenicity among human mtDNA polymorphisms}

A subset of substitutions currently annotated as 'polymorphic' in the MITOMAP database were distinguished by very low TSS scores at the relevant alignment position, 
suggesting that mutations at those positions could, in fact, be deleterious. Moreover, when considering polymorphisms, the absence of IIDSs within the mammalian phylogenetic tree that would match particular human variations suggested that additional pathogenicity may exist among human mtDNA substitutions currently considered polymorphic. In order to explore whether additional pathogenic mutations might indeed be discovered among variants currently tabulated as 'polymorphic,' we tested whether protein polymorphisms tabulated in MITOMAP that cannot be directly linked to the human reference character along internal edges of the mammalian phylogenetic tree are under purifying selection. Selection was assessed by determining the number of full-length mtDNA samples found in GenBank (Benson et al, 2013) that were assigned within MITOMAP to a given variant. Measuring selection by assessment of population frequencies can be problematic due to potential sampling biases and population bottlenecks (Auer \& Lettre, 2015), timing of mutation arrival within an expanding population (Luria \& Delbrück, 1943; Rosche \& Foster, 2000), and the divergent nucleotide- and strand-specific mutational propensities of mtDNA (Tanaka \& Ozawa, 1994; Faith \& Pollock, 2003; Reyes et al, 1998). Even so, the distribution of variant frequencies among full-length sequences in GenBank was strikingly different for those mutations for which an IIDS could be identified in our mammalian trees of proteins (Figure 3B), and even tRNAs (Figure 3C), when compared to those for which an IIDS could not be identified. These results indicate that many human mtDNA protein and tRNA variants currently considered to be polymorphic are, in fact, harmful, and these findings further validate the development of the IIDS as a feature useful for determining variant pathogenicity.

\section{A support vector machine predicts harmful mtDNA variants}

Given the clear presence of deleterious substitutions among so far uncharacterized variants, we sought a high-throughput method that could, with confidence, identify these potentially deleterious substitutions. Toward this goal, we turned to machine learning. Specifically, we deployed a support vector machine (SVM) (Cortes \& Vapnik, 1995), designed to minimize risk of misclassification and able to take advantage of non-linear features (Bhavsar \& Ganatra, 2012), to predict the risk of mtDNA variants using our conservation features. Positive training sets for our SVM consisted of protein or tRNA mutations annotated as confirmed pathogenic alterations within the MITOMAP database (Lott et al, 2013). Negative training sets included variants annotated as polymorphic in MITOMAP and also characterized by the highest counts of homoplasmic alleles within HelixMTdb, a database emerging from a survey of nearly 200,000 human samples (Bolze et al, 2019). Beyond these training variants, our total set of variants for prediction included substitutions tabulated in MITOMAP, substitutions indexed in HelixMTdb, and simulated substitutions from the reference sequence not yet encountered in the clinic. Analyzed positions were gapped within less than or equal to $1.5 \%$ of analyzed sequences. As SVM features, we exploited TSS, ISS, and IIDS. However, since SVMs may beneficially 
incorporate features, even with theoretical shortcomings, in unanticipated ways, we also expanded our feature set to include other features related to conservation. These additional features included the number of characters found at alignment positions (internal edges and all edges), whether the mutant character is ever found at internal nodes, and positional Shannon entropy.

For variants found within protein-coding genes, our SVM approach clearly permitted a robust and global prediction of which polymorphisms not yet classified as pathogenic may cause or contribute to disease (Figure 4A and Table S1). Importantly, when examining the behavior of protein-coding variants within our negative training sets, only one false-positive was obtained. Furthermore, few confirmed pathogenic mutations were found among the predicted negative set. The proficiency of our model in correctly identifying true positives in the training set without a corresponding loss in specificity is highlighted by the associated Receiver Operating Characteristic (ROC) curve [area under the receivership curve (AUC), 0.96] (Figure S3A). The Matthews Correlation Coefficient (MCC), reflecting the overall performance of our classifier (Guilford, 1954; Chicco \& Jurman, 2020), was 0.89, indicating a model with high proficiency at separating our positive and negative training sets. Accuracy for our model was calculated to be 0.95 , precision was 0.97 , sensitivity was 0.90 , specificity was 0.98 , and our F-score was 0.94 , all of which are outcomes that also suggest outstanding classifier performance on variation within mitochondria-encoded polypeptides. Of features assessed by our SVM, all of which are related to site conservation among mammals, IIDS stood out as dominant in providing predictive value (Figure S3B).

To further validate our approach, we turned to the thousands of uncharacterized mtDNA changes encountered during generation of the HelixMTdb. Data presented above (Figure 3B and 3C) and previous studies (Ye et al, 2014; Elliott et al, 2008; Wei \& Chinnery, 2020) support the idea that pathogenic alleles reside among the set of uncharacterized changes and mutations currently annotated as polymorphic. Moreover, frequent clinical encounters with a variant observed in a heteroplasmic state (not consisting of $100 \%$ of the molecules in a cell) can often be a signal of pathogenicity (Stewart \& Chinnery, 2015; Hahn \& Zuryn, 2019). Therefore, we reasoned that by first separating unannotated variants by SVM prediction class, then comparing the fraction of instances that each allele within that class was determined to be heteroplasmic within an individual, we might assess our prediction capability. We note that heteroplasmy was not a feature used for development of our prediction model. Upon analysis, heteroplasmy profiles were quite clearly distinct (Figure 4B) when comparing those variants that we predict to be harmful and those variants that we predict to be benign, and we obtained a Kolmogorov-Smirnov D statistic of 0.471 , suggesting that our methodology has quite successfully revealed alleles in protein-coding genes that are likely to cause metabolic disease. We have named our approach MitoCAP, for "Mitochondrial Disease Predictions Using hhanges Across Phylogeny". 
Other classifiers provide their own predictions, or otherwise provide the opportunity to predict, whether some or all of the potential changes in mtDNA-encoded proteins may be deleterious. We selected several of these methods for comparison with MitoCAP. We focused our attention primarily upon two recently developed and mitochondria-specific classifiers: Mitoclass (Martín-Navarro et al, 2017) and the meta-classifier Mitlmpact (Castellana et al, 2015). We also included two other tools that allow batch submission of substitution input, PolyPhen-2 (Adzhubei et al, 2013) and PROVEAN (Choi et al, 2012), promoting further evaluation of mitochondria-specific classifiers. Finally, we chose PantherPSEP (Tang \& Thomas, 2016b) which, like MitoCAP, takes advantage of phylogenetic tree information while making predictions. These different classifiers do include some convergent concepts within their prediction schemes. However, we expected that our use of novel phylogenetic and conservation features would lead to tangible improvement in the prediction of mtDNA variant pathogenicity.

First, we tested how the compared methods would fare against the training set that was used to build our own prediction model for variants in protein-coding genes. It may be challenging, in an unbiased manner, to compare metrics of prediction success that we obtained using our own training sets to those achieved by other methods using our training sets. However, selection of variants for inclusion within our training sets was based upon conservative measures of pathogenicity or benignancy, and not upon our own interpretation of individual variant hazard. Therefore, we should expect other classifiers to also perform well on our training set. Of the six methods compared, the two methods specifically designed to assess variants within mitochondrial protein coding genes, Mitlmpact and Mitoclass, performed second and third best on our training set, as reflected by MCC values. These classifiers were followed by PROVEAN, PolyPhen-2, and Panther-PSEP (Figure 4C). MitoCAP also scored best against our training set when considering most auxiliary measures of prediction proficiency (Figure S4).

We further explored the magnitude by which the underlying prediction models might differ from one another. To further investigate this possibility, we first plotted the level of agreement between MitoCAP other methods when assessing all classified variants, and we noted a pronounced lack of overlap between our MitoCAP predictions and the predictions of other methods (Figure 4D). For example, Mitlmpact appears to predict that a substantial number of alleles that we predict as pathogenic are actually innocuous. In contrast, PantherPSEP predicts that a pronounced number of alleles that we call benign are actually deleterious.

In order to more quantitatively assess how similar each pair of prediction outcomes may be to one another, we next applied MCC calculations as a way to measure prediction correspondence. During these analyses (Figure 4E), the maximum MCC value calculated during comparisons between methods was 0.61 , obtained during comparison of MitoCAP 
and PROVEAN, and a similar score of 0.60 was the outcome of comparing the MitoCAP and Mitoclass predictors. All other comparisons provided MCC values of 0.5 or (sometimes substantially) less. Therefore, MitoCAP seems to provide predictions for unannotated variants that are most in accordance with, but not identical to, those produced by PROVEAN and Mitoclass.

But which of these prediction approaches is most likely to appropriately characterize unannotated variants? When heteroplasmy data for unannotated variants in HelixMTdb are analyzed for other prediction methods (Figure S5), as performed above for MitoCAP, MitoCAP best separated variants into classes with different heteroplasmy propensities and achieved the highest Kolmogorov-Smirnov D score (Figure 4F). Consequently, our prediction method appears to be the most successful among the tested classifiers in identifying harmful mtDNA variants within protein-coding genes.

For mitochondria-encoded tRNAs, notable separation between positive and negative training sets (Figure S6A and Table S2) was also achieved by MitoCAP. When considering our tRNA training set, the MitoCAP MCC value was 0.82 , accuracy was 0.91 , precision was 0.86 , sensitivity was 0.95 , specificity was 0.88 , and the F-score was 0.90 . The strong performance of our classifier is exemplified by the ROC curve (Figure S3C) and the associated AUC of 0.94 . The number of characters seen at internal nodes for positions of interest, as well as the TSS and ISS, appeared to play the strongest role during development of the SVM prediction model (Figure S3D). When samples outside of our training set from our two predictive classes were plotted for heteroplasmy classification frequency in HelixMTdb, we again noted a significant difference in the two distributions (Figure S6B), indicating successful prediction of previously unanticipated pathogenicity. When compared to two other methods of tRNA variant pathogenicity prediction, PON-mt-tRNA (Niroula \& Vihinen, 2016) and MitoTIP (Sonney et al, 2017), the MitoTIP classifier performed better when considering the MitoCAP training set (Figure S6C and Figure S7). All three methods tested appear to diverge substantially from one another in their pathogenicity predictions (Figure S6D and Figure S6E). MitoTIP most distinctly separated distributions of heteroplasmy frequency within different predictive classes of unannotated variants (Figure S6F and Figure S8). However, superior separation of heteroplasmy distributions by MitoTIP may not be surprising, since MitoTIP incorporates whether variants have been encountered as heteroplasmic during its classifications, while MitoCAP does not.

Taken together, our analyses indicate that MitoCAP appears to be the most proficient among the compared methods in predicting pathogenicity of variants in mtDNA-encoded proteins, while alternative methods may outperform MitoCAP during classification of tRNA variants. 


\section{DISCUSSION}

We describe here a methodology that allows improved quantification of the relative conservation of sites within and between genes, RNAs, and proteins. TSS and ISS values provide a measure of conservation that can minimize errors in conservation calculation that result from sampling biases. Importantly, these scores are of theoretically unlimited dynamic range and will benefit from the continuous expansion of available sequence information. Even nearly identical sequences can be utilized by our approach, allowing for an everincreasing input dataset that can be deployed toward calculation of site-specific conservation.

Beyond the development of a novel quantitative measure of conservation, we also use ancestral predictions to generate IIDSs - a binary read-out of site-specific substitution information. Researchers often consult generalized substitution matrices when predicting whether a change may be harmful or not (Dayhoff et al, 1978; Jones et al, 1992), yet amino acid exchangeability matrices change when moving across clades (Zou \& Zhang, 2019), and successful substitution of any given character occurs in the context of a very specific local environment (Zuckerkandl \& Pauling, 1965; Tang \& Thomas, 2016a). By mapping substitutions to phylogenetic tree edges, ample sequence data can allow identification of IIDSs within the context of a particular macromolecular position, thereby improving prediction of variant pathogenicity. We note that focusing upon IIDSs, rather than the simple presence or absence of a character at a site, can indirectly integrate information about potential epistatic interactions that permit or block a substitution from being successfully established within a lineage.

Protein variants of confirmed pathogenicity were clearly characterized by low substitution scores and by an absence of IIDSs. But how should one weigh these and other available factors when classifying, in an unbiased and high-throughput manner, other variants already encountered or waiting to be discovered among human mtDNAs? To address this question, we deployed a machine learning approach, trained on existing data present in the MITOMAP database, toward determination of mtDNA mutation pathogenicity. Our apparent false-positive rate for protein coding variants appears to be extremely low, which is essential when avoiding the potential psychological effects of a false-positive test (Stewart-Brown \& Farmer, 1997; Committee on Bioethics, 2001). Moreover, metrics of prediction capability for mitochondrial amino acid substitutions indicate very strong proficiency at pathogenicity prediction (MCC of 0.89 ), and specificity and sensitivity metrics resulting from our SVM predictions appear exemplary (AUC > 0.96). Importantly, comparison of multiple approaches by measuring the difference in heteroplasmy propensity, a common indicator of pathogenicity (Ye et al, 2014), within different predicted classes suggests that we can successfully outperform other methods specifically designed to classify 
mitochondrial variants, as well as several approaches focused more generally upon protein substitutions.

The MitoCAP predictions that we provide allow for improved comprehension of which mtDNA variants identified within a patient may be linked to mitochondrial disease. Our approach also allows researchers focusing upon the fundamental aspects of mitochondrial disease to rapidly prioritize variants identified in the clinic for directed study.

\section{mtDNA variants predicted to be pathogenic may lead to cryptic and age-related mitochondrial disease}

Our results are congruent with earlier analyses suggesting that harmful mtDNA substitutions may be common within the human population (Ye et al, 2014; Elliott et al, 2008; Nachman et al, 1996). So why have so many of these deleterious changes not yet been classified as pathogenic within the clinic?

First, if a mutation is relatively common within the population, clinicians may inappropriately determine that the mtDNA variant is unlinked to patient symptoms (Whiffin et al, 2017). However, population frequency is not currently, at least when taken in isolation, a highly reliable predictor of clinical outcome, since population counts require substantial correction for strand- and nucleotide-specific mtDNA mutational biases (Tanaka \& Ozawa, 1994; Faith \& Pollock, 2003; Reyes et al, 1998), and sampling biases are typically a hazard when carrying out population-wide studies (Zuk et al, 2014; Chheda et al, 2017; Keinan \& Clark, 2012; Landry et al, 2018).

Second, deleterious mtDNA variants often must rise beyond a certain threshold among the hundreds of mtDNA molecules potentially resident within a cell before overt symptoms can manifest (Stewart \& Chinnery, 2015; Russell et al, 2020). Concordantly, our data suggest a strong propensity for heteroplasmy in the set of substitutions that we predict to be pathogenic, but are not yet clinically annotated as disease-associated. Differential distribution of mtDNA variants within a carrier, either established during development or resulting from bottleneck effects in renewable and non-mitotic tissues (Zhang et al, 2018; Stewart \& Chinnery, 2015), may generate clones with a high proportion of deleterious mutations and may lead to complex, tissue-specific outcomes (Greaves et al, 2006; Nekhaeva et al, 2002; Bratic \& Larsson, 2013; Fayet et al, 2002). Moreover, the phenomena described above may lead to age-related symptoms not easily classified as mitochondrial disease, since even relatively common mtDNA sequence variants have been suggested to contribute to diseases like diabetes, heart disease, and cancer (Chinnery \& Gomez-Duran, 2018; Marom et al, 2017; Wei et al, 2017). We are certainly tantalized by the prospect that pathogenic variants illuminated by our approach might impinge upon human lifespan. 


\section{METHODOLOGY}

Mitochondrial DNA sequence acquisition and conservation analysis

Mammalian mtDNA sequences were retrieved from the National Center for Biotechnology Information database of organelle genomes

(https://www.ncbi.nlm.nih.gov/genome/browse\#!/organelles/ on September 26, 2019). These 1184 mammalian mtDNA genomes were aligned using MAFFT on the 'auto' setting (Katoh \& Standley, 2013). Four sequences that were egregiously misaligned were removed, and MAFFT alignment with the 'auto' setting was carried out again. Individual gene sequences were extracted from these alignments, based upon the annotated human mtDNA (NC_012920.1). After gap removal, translation of protein coding genes was performed using the vertebrate mitochondrial codon table in AliView (Larsson, 2014). MAFFT alignments of each gene product were performed using the G-INS-i iterative refinement method, then individual concatenates for each species were generated from protein coding sequences, tRNAs, and rRNAs. Duplicates were removed from the protein, tRNA, and rRNA concatenates using seqkit v0.10.2 (Shen et al, 2016).

Maximum likelihood trees for each molecule class concatenate were built using FastTreeMP (Price et al, 2010) with four subtree-prune-regraft moves, two rounds of branch length optimization, slow nearest neighbor interchange, and by use of a generalized timereversible model. Next, ancestral prediction was carried out using the PAGAN package (Löytynoja et al, 2012), with concatenated alignments and phylogenetic trees used as input. The PAGAN output was then analyzed using "binary-table-by-edges-v2.2" and "addconvention-to-binarytable-v1.1.py" (https://github.com/corydunnlab/hummingbird) (Dunn et al, 2020) to allow for a sum of substitutions at alignment positions encoded by human mtDNA. All fluctuating edges were extracted using "report-on-F-values-v1.1" (https://github.com/corydunnlab/hummingbird). Correspondence files for the human mtDNA (NC_012920.1) convention and alignment positions were generated using "extractcorrespondence-for-merged-alignment-v.1.1.py", followed by the construction of the lookup tables of amino acid direct substitution and presence using "direct-subst-lookup-tableproteins-v.1.1.py" and "AA-presence-lookup-table-v.1.1.py", respectively (https://github.com/corydunnlab/Edge_mapping_conservation). For IIDS determination, only edges with standard characters at both connected nodes were considered. For TSS and ISS calculation, the PAGAN output files for tRNAs and rRNAs were, before inferring a substitution, processed so that each ambiguous IUPAC nucleotide code was replaced by one of the possible standard nucleotides represented by that IUPAC code within the entire tree at that given position. For positions that contained only N's and gaps, N's were replaced by gaps. Replacement of ambiguous characters with standard characters was carried out using using script "replace-nonstandard.py" (https://github.com/corydunnlab/Edge_mapping_conservation), and the standard nucleotide 
was picked at random from available standard characters at a given alignment position using the random.choice() function of the Python random module. Gap percentage for each position was calculated using trimAl v1.4.rev22 (Capella-Gutiérrez et al, 2009) on alignments removed of predicted sequences for internal nodes. Shannon entropy was calculated using the 'Shannon' script (https://gist.github.com/jrjhealey/), also on alignments removed of predicted sequences for internal nodes. Custom scripts were used to merge various data tables. Software generated within the context of this study was written using Python 2.7.

Statistical testing and graph production were performed using Prism 8.4.3 (https://www.graphpad.com).

mtDNA variant database utilization

MITOMAP data used in this study (Lott et al, 2013) were downloaded on October 1, 2019. HelixMTdb data used in this study (Bolze et al, 2019) were downloaded on October 15,2019 . Only substitutions were analyzed within the context of this study; insertions and deletions were not analyzed.

Support vector machine classification

Our SVM classifier (Cortes \& Vapnik, 1995) was developed using the R-language (https://www.R-project.org/) package e1071 (Meyer et al, 2014), for which the implementation is based on libsvm (Chang \& Lin, 2011). A supervised binary classification model was built using positive and negative training sets. Each negative training set included those alleles with the highest number of homoplasmic samples in HelixMTdb and also annotated as a polymorphism within MITOMAP. For proteins, the negative training sets consisted of 50 mtDNA substitutions (encoding 51 protein variants) from the reference sequence. For tRNAs, the negative training sets also consisted of 50 substitutions. Positive training sets consisted of variants confirmed as pathogenic in MITOMAP on the date the database was queried. The positive training set for mtDNA-encoded proteins consisted of 39 mtDNA substitutions (encoding 40 protein variants), and the positive training set for mtDNA-encoded tRNAs consisted of 39 substitutions. Predictions were made for protein and tRNA sites gapped at $1.5 \%$ or less within our alignments. Decision values are related to, but do not directly represent, scalar distance from variant points to SVM margins (Sanz et al, 2018).

The best cost and gamma parameters for the classification model were selected based on majority voting of 100 -searches of $2^{\wedge}(-8: 8)$ grid surface, using 'tune.svm' function of e1071 [kernel = 'radial', type = 'C-classification', scale $=$ TRUE, tunecontrol = tune.control (cross $=5$, nrepeat $=10$ )]. The classification model was subsequently built and trained using the optimized [gamma, cost] parameters of [0.25, 0.0625] for proteins (Figure S9A) and 
$[0.0078125,256]$ for tRNAs (Figure S9B), and subsequently used to predict the pathogenicity of the training and test set variants. Features were scaled internally during training and when applying the model to predict the test set. Predictions for the ROC curve were collected using 'mining' function of the rminer package (Cortez, 2015), with the optimized parameters during 10 runs of 5-fold cross-validation [model="ksvm", task = "prob", method = c("kfold", $5)$, Runs = 10]. Feature importance was measured using the 'Importance' function from rminer (Cortez \& Embrechts, 2013), which was run 10 times (method = "DSA").

Due to the relatively limited number of variants confirmed to be pathogenic or benign that one might include in a balanced training set, we also separately evaluated the robustness of our classifier using multiple runs of k-fold cross-validation with varying values of $\mathrm{k}$. During these experiments, the complete list of our training set was divided into k parts, the classifier was trained on the $(\mathrm{k}-1)$ parts and the remaining part was used to evaluate the classifier performance. The k-fold cross-validation runs were performed with the optimized cost and gamma parameters given above. For both protein (Figure S9C) and tRNA (Figure S9D) training sets, 2-fold, 3.4-fold and 5-fold cross-validation was run 10 times, corresponding to setting 50\%, 30\% and $20 \%$, respectively, of the training set aside for testing, then training the classifier with the remaining variants. In each run, mean performance metrics were collected by the 'mining' function over 10 repeats of the respective k-fold cross-validation [model = "ksvm", task = "prob", method = c("kfold", ${ }^{*}$ variable*), runs $=10$ ). Predictions for the set reserved for testing were then used to calculate performance metrics. The performance metrics MCC, Accuracy (ACC), True Positive Rate (TPR), True Negative Rate (TNR) and Area Under the ROC Curve (AUC) were calculated using 'mmetric' of the rminer package.

Comparison of selected, alternative prediction methods with MitoCAP

Pathogenicity predictions for our training and test set variants were compared to predictions made by PolyPhen-2 (Adzhubei et al, 2013), PROVEAN (Choi et al, 2012), Panther-PSEP (Tang \& Thomas, 2016b), Mitoclass (Martín-Navarro et al, 2017) and Mitlmpact (Castellana et al, 2015). PolyPhen-2 predictions were retrieved from its web server in batch query mode with the HimDiv classifier and default parameters. Similarly, PROVEAN predictions were retrieved from the web server for 'PROVEAN Protein Batch' tool for human proteins. For Panther-PSEP, variant queries for each of the 13 mitochondria-encoded proteins were generated and submitted, along with the protein sequence, to the web server. Mitoclass predictions were retrieved from the supplemental data of its publication (MartínNavarro et al, 2017). For the meta-classifier employing its own SVM, Mitlmpact, the latest predictions (Mitlmpact 3D 3.0.5) were used. For comparison with PON-mt-tRNA (Niroula \& Vihinen, 2016), tested variants were uploaded to the associated web server, and output was processed according to the mtDNA strand upon which the tRNA is encoded. The most 
recent MitoTIP (Sonney et al, 2017) predictions were published on April 27, 2020 and were downloaded directly from the MITOMAP server.

For PolyPhen-2 and Panther-PSEP, predictions 'possibly damaging' and 'probably damaging' were considered as 'pathogenic'. For MitoTIP predictions, 'likely pathogenic' and 'possibly pathogenic' were collapsed to 'pathogenic' during comparisons, while 'possibly benign' and 'likely benign' were reduced to 'benign'. Similarly, for PON-mt-tRNA annotations, 'pathogenic' and 'likely pathogenic' were reduced to 'pathogenic', while 'likely neutral' and 'neutral' annotations were considered as 'benign'. Since Mitlmpact is a metaclassifier working on mtDNA sequence, it can produce two different pathogenicity predictions for two nucleotide changes leading to the same amino acid change. Such instances, where the same amino acid change is labeled both as 'pathogenic' and 'neutral' by Mitlmpact, were classified as 'contradictory' and not further processed during analyses.

\section{ACKNOWLEDGEMENTS}

C.D.D. is funded by a ERC Starter Grant (RevMito 637649), by the Sigrid Jusélius Foundation, and by the Academy of Finland (331556). V.O.P. is supported by the Academy of Finland (289737, 314672, and 330255), U.S. National Institute of General Medical Sciences (1R01GM132649-01), the Sigrid Jusélius Foundation, and by the Runar Bäckström Foundation. We thank Vivek Sharma, Tamara Somborac, Svetlana Konovalova, and Gülayşe Ince Dunn for helpful manuscript comments.

\section{AUTHOR CONTRIBUTIONS}

B.A.A. developed software, analyzed data, and edited the manuscript. P.O.C. and V.O.P. analyzed data and edited the manuscript. C.D.D. conceived of the classification approach, supervised the project, analyzed data, prepared figures, and wrote the manuscript.

\section{DISCLOSURES}

C.D.D. is managing director, and B.A.A., and P.O.C. are members, of Primal Predictions LLC, a firm developing approaches to variant pathogenicity prediction.

\section{SUPPLEMENTAL TABLE LEGENDS}

Table S1: SVM output analyzing protein-encoding variants found in the MITOMAP and HelixMTdb by use of mammalian sequence data. Details of SVM analyses and features are found within the methodology section. 
Table S2: SVM output analyzing tRNA-encoding variants found in the MITOMAP and HelixMTdb by use of mammalian sequence data. Details of SVM analyses and features are found within the methodology section.

\section{REFERENCES}

Adzhubei I, Jordan DM \& Sunyaev SR (2013) Predicting functional effect of human missense mutations using PolyPhen-2. Curr. Protoc. Hum. Genet. Chapter 7: Unit7.20

Alston CL, Rocha MC, Lax NZ, Turnbull DM \& Taylor RW (2017) The genetics and pathology of mitochondrial disease. J. Pathol. 241: 236-250

Auer PL \& Lettre G (2015) Rare variant association studies: considerations, challenges and opportunities. Genome Med. 7: 16

Benson DA, Cavanaugh M, Clark K, Karsch-Mizrachi I, Lipman DJ, Ostell J \& Sayers EW (2013) GenBank. Nucleic Acids Res. 41: D36-42

Bhavsar H \& Ganatra A (2012) A comparative study of training algorithms for supervised machine learning. International Journal of Soft Computing and Engineering (IJSCE) 2: 2231-2307

Blell M \& Hunter MA (2019) Direct-to-Consumer Genetic Testing's Red Herring: 'Genetic Ancestry' and Personalized Medicine. Front. Med. 6: 48

Bolze A, Mendez F, White S, Tanudjaja F, Isaksson M, Rashkin M, Bowes J, Cirulli ET, Metcalf WJ, Grzymski JJ, Lee W, Lu JT \& Washington NL (2019) Selective constraints and pathogenicity of mitochondrial DNA variants inferred from a novel database of 196,554 unrelated individuals. bioRxiv: 1151

Boulet L, Karpati G \& Shoubridge EA (1992) Distribution and threshold expression of the tRNA(Lys) mutation in skeletal muscle of patients with myoclonic epilepsy and raggedred fibers (MERRF). Am. J. Hum. Genet. 51: 1187-1200

Bratic A \& Larsson N-G (2013) The role of mitochondria in aging. J. Clin. Invest. 123: 951957

Capella-Gutiérrez S, Silla-Martínez JM \& Gabaldón T (2009) trimAl: a tool for automated alignment trimming in large-scale phylogenetic analyses. Bioinformatics 25: 1972-1973

Castellana S, Rónai J \& Mazza T (2015) MitImpact: an exhaustive collection of pre-computed pathogenicity predictions of human mitochondrial non-synonymous variants. Hum.

Mutat. 36: E2413-22

Chang C-C \& Lin C-J (2011) LIBSVM: A library for support vector machines. ACM transactions on intelligent systems and technology (TIST) 2: 27

Chen L, Liu P, Evans TC Jr \& Ettwiller LM (2017) DNA damage is a pervasive cause of 
sequencing errors, directly confounding variant identification. Science 355: 752-756

Chheda H, Palta P, Pirinen M, McCarthy S, Walter K, Koskinen S, Salomaa V, Daly M, Durbin R, Palotie A, Aittokallio T \& Ripatti S (2017) Whole-genome view of the consequences of a population bottleneck using 2926 genome sequences from Finland and United Kingdom. Eur. J. Hum. Genet. 25: 477-484

Chicco D \& Jurman G (2020) The advantages of the Matthews correlation coefficient (MCC) over F1 score and accuracy in binary classification evaluation. BMC Genomics 21: 6

Chinnery PF \& Gomez-Duran A (2018) Oldies but Goldies: mtDNA Population Variants and Neurodegenerative Diseases. Front. Neurosci. 12: 682

Choi Y, Sims GE, Murphy S, Miller JR \& Chan AP (2012) Predicting the functional effect of amino acid substitutions and indels. PLoS One 7: e46688

Committee on Bioethics (2001) Ethical issues with genetic testing in pediatrics. Pediatrics 107: 1451-1455

Cortes C \& Vapnik V (1995) Support-vector networks. Mach. Learn. 20: 273-297

Cortez P (2015) A tutorial on using the rminer R package for data mining tasks Universidade do Minho. Escola de Engenharia (EEng) Available at: https://repositorium.sdum.uminho.pt/handle/1822/36210

Cortez P \& Embrechts MJ (2013) Using sensitivity analysis and visualization techniques to open black box data mining models. Inf. Sci. 225: 1-17

Cui R, Medeiros T, Willemsen D, lasi LNM, Collier GE, Graef M, Reichard M \& Valenzano DR (2019) Relaxed Selection Limits Lifespan by Increasing Mutation Load. Cell 178: 385399.e20

Dayhoff M, Schwartz R \& Orcutt B (1978) 22 a model of evolutionary change in proteins. In Atlas of protein sequence and structure pp 345-352. National Biomedical Research Foundation Silver Spring MD

Dunn CD, Akpınar BA \& Sharma V (2020) An Unusual Amino Acid Substitution Within Hummingbird Cytochrome c Oxidase Alters a Key Proton-Conducting Channel. G3 10: 2477-2485

Elliott HR, Samuels DC, Eden JA, Relton CL \& Chinnery PF (2008) Pathogenic mitochondrial DNA mutations are common in the general population. Am. J. Hum. Genet. 83: 254-260

Faith JJ \& Pollock DD (2003) Likelihood analysis of asymmetrical mutation bias gradients in vertebrate mitochondrial genomes. Genetics 165: 735-745

Fayet G, Jansson M, Sternberg D, Moslemi AR, Blondy P, Lombès A, Fardeau M \& Oldfors A (2002) Ageing muscle: clonal expansions of mitochondrial DNA point mutations and deletions cause focal impairment of mitochondrial function. Neuromuscul. Disord. 12: 
484-493

da Fonseca RR, Johnson WE, O’Brien SJ, Ramos MJ \& Antunes A (2008) The adaptive evolution of the mammalian mitochondrial genome. BMC Genomics 9: 119

Gorman GS, Schaefer AM, Ng Y, Gomez N, Blakely EL, Alston CL, Feeney C, Horvath R, YuWai-Man P, Chinnery PF, Taylor RW, Turnbull DM \& McFarland R (2015) Prevalence of nuclear and mitochondrial DNA mutations related to adult mitochondrial disease. Ann.

Neurol. 77: 753-759

Greaves LC, Preston SL, Tadrous PJ, Taylor RW, Barron MJ, Oukrif D, Leedham SJ, Deheragoda M, Sasieni P, Novelli MR, Jankowski JAZ, Turnbull DM, Wright NA \& McDonald SAC (2006) Mitochondrial DNA mutations are established in human colonic stem cells, and mutated clones expand by crypt fission. Proc. Natl. Acad. Sci. U. S. A. 103: 714-719

Guilford JP (1954) Psychometric methods, 2nd ed. 2: 597

Hahn A \& Zuryn S (2019) The Cellular Mitochondrial Genome Landscape in Disease. Trends Cell Biol. 29: 227-240

Hill GE, Havird JC, Sloan DB, Burton RS, Greening C \& Dowling DK (2019) Assessing the fitness consequences of mitonuclear interactions in natural populations. Biol. Rev. Camb. Philos. Soc. 94: 1089-1104

lantorno S, Gori K, Goldman N, Gil M \& Dessimoz C (2014) Who watches the watchmen? An appraisal of benchmarks for multiple sequence alignment. Methods Mol. Biol. 1079: 5973

Jones DT, Taylor WR \& Thornton JM (1992) The rapid generation of mutation data matrices from protein sequences. Comput. Appl. Biosci. 8: 275-282

Katoh K \& Standley DM (2013) MAFFT multiple sequence alignment software version 7: improvements in performance and usability. Mol. Biol. Evol. 30: 772-780

Kawrykow A, Roumanis G, Kam A, Kwak D, Leung C, Wu C, Zarour E, Phylo players, Sarmenta L, Blanchette M \& Waldispühl J (2012) Phylo: a citizen science approach for improving multiple sequence alignment. PLoS One 7: e31362

Keinan A \& Clark AG (2012) Recent explosive human population growth has resulted in an excess of rare genetic variants. Science 336: 740-743

Khan S, Ince-Dunn G, Suomalainen A \& Elo LL (2020) Integrative omics approaches provide biological and clinical insights: examples from mitochondrial diseases. J. Clin. Invest. 130: $20-28$

Kimura M (1985) The role of compensatory neutral mutations in molecular evolution. J. Genet. 64: 7 
Kondrashov AS, Sunyaev S \& Kondrashov FA (2002) Dobzhansky-Muller incompatibilities in protein evolution. Proc. Natl. Acad. Sci. U. S. A. 99: 14878-14883

Landry LG, Ali N, Williams DR, Rehm HL \& Bonham VL (2018) Lack Of Diversity In Genomic Databases Is A Barrier To Translating Precision Medicine Research Into Practice. Health Aff. 37: 780-785

Larsson A (2014) AliView: a fast and lightweight alignment viewer and editor for large datasets. Bioinformatics 30: 3276-3278

Lott MT, Leipzig JN, Derbeneva O, Xie HM, Chalkia D, Sarmady M, Procaccio V \& Wallace DC (2013) mtDNA Variation and Analysis Using Mitomap and Mitomaster. Curr. Protoc. Bioinformatics 44: 1.23.1-26

Löytynoja A, Vilella AJ \& Goldman N (2012) Accurate extension of multiple sequence alignments using a phylogeny-aware graph algorithm. Bioinformatics 28: 1684-1691

Luria SE \& Delbrück M (1943) Mutations of Bacteria from Virus Sensitivity to Virus Resistance. Genetics 28: 491-511

Maklakov AA, Rowe L \& Friberg U (2015) Why organisms age: Evolution of senescence under positive pleiotropy? Bioessays 37: 802-807

Marini NJ, Thomas PD \& Rine J (2010) The use of orthologous sequences to predict the impact of amino acid substitutions on protein function. PLoS Genet. 6: e1000968

Marom S, Friger M \& Mishmar D (2017) MtDNA meta-analysis reveals both phenotype specificity and allele heterogeneity: a model for differential association. Sci. Rep. 7: 43449

Martín-Navarro A, Gaudioso-Simón A, Álvarez-Jarreta J, Montoya J, Mayordomo E \& RuizPesini E (2017) Machine learning classifier for identification of damaging missense mutations exclusive to human mitochondrial DNA-encoded polypeptides. BMC Bioinformatics 18: 158

Matilainen O, Quirós PM \& Auwerx J (2017) Mitochondria and Epigenetics - Crosstalk in Homeostasis and Stress. Trends Cell Biol. 27: 453-463

Medawar PB (1952) An Unsolved Problem of Biology: An Inaugural Lecture Delivered at University College, London, 6 December, 1951 H.K. Lewis and Company

Meyer D, Dimitriadou E, Hornik K, Weingessel A, Leisch F, Chang CC \& Lin CC (2014) e1071: Misc functions of the Department of Statistics (e1071), TU Wien. $R$ package version 1: Available at: http://www2.uaem.mx/r-mirror/web/packages/e1071/

Nabholz B, Ellegren H \& Wolf JBW (2013) High levels of gene expression explain the strong evolutionary constraint of mitochondrial protein-coding genes. Mol. Biol. Evol. 30: 272284 
Nachman MW (1998) Deleterious mutations in animal mitochondrial DNA. Genetica 102-103: 61-69

Nachman MW, Brown WM, Stoneking M \& Aquadro CF (1996) Nonneutral mitochondrial DNA variation in humans and chimpanzees. Genetics 142: 953-963

Nekhaeva E, Bodyak ND, Kraytsberg Y, McGrath SB, Van Orsouw NJ, Pluzhnikov A, Wei JY, Vijg J \& Khrapko K (2002) Clonally expanded mtDNA point mutations are abundant in individual cells of human tissues. Proc. Natl. Acad. Sci. U. S. A. 99: 5521-5526

Niroula A \& Vihinen M (2016) PON-mt-tRNA: a multifactorial probability-based method for classification of mitochondrial tRNA variations. Nucleic Acids Res. 44: 2020-2027

Phillips KA, Deverka PA, Hooker GW \& Douglas MP (2018) Genetic Test Availability And Spending: Where Are We Now? Where Are We Going? Health Aff. 37: 710-716

Pirastu N, Cordioli M, Nandakumar P, Mignogna G, Abdellaoui A, Hollis B, Kanai M, Rajagopal VM, Della Briotta Parolo P, Baya N, Carey C, Karjalainen J, Als TD, Van der Zee MD, Day FR, Ong KK, Finngen Study, Me Research Team, Consortium iPSYCH, Morisaki T, et al (2020) Genetic analyses identify widespread sex-differential participation bias. bioRxiv: 2020.03.22.001453 Available at:

https://www.biorxiv.org/content/10.1101/2020.03.22.001453v1 [Accessed March 24, 2020]

Price MN, Dehal PS \& Arkin AP (2010) FastTree 2--approximately maximum-likelihood trees for large alignments. PLoS One 5: e9490

Raychaudhuri S (2011) Mapping rare and common causal alleles for complex human diseases. Cell 147: 57-69

Reyes A, Gissi C, Pesole G \& Saccone C (1998) Asymmetrical directional mutation pressure in the mitochondrial genome of mammals. Mol. Biol. Evol. 15: 957-966

Rosche WA \& Foster PL (2000) Determining Mutation Rates in Bacterial Populations. Methods 20: 4-17

Russell OM, Gorman GS, Lightowlers RN \& Turnbull DM (2020) Mitochondrial Diseases: Hope for the Future. Cell 181: 168-188

Sanz H, Valim C, Vegas E, Oller JM \& Reverter F (2018) SVM-RFE: selection and visualization of the most relevant features through non-linear kernels. BMC Bioinformatics 19: 432

Shen W, Le S, Li Y \& Hu F (2016) SeqKit: A Cross-Platform and Ultrafast Toolkit for FASTA/Q File Manipulation. PLoS One 11: e0163962

Smith DR (2019) Revisiting published genomes with fresh eyes and new data: Revising old sequencing data can yield unexpected insights and identify errors. EMBO Rep. 20: e49482 
Sonney S, Leipzig J, Lott MT, Zhang S, Procaccio V, Wallace DC \& Sondheimer N (2017) Predicting the pathogenicity of novel variants in mitochondrial tRNA with MitoTIP. PLoS Comput. Biol. 13: e1005867

Stewart-Brown S \& Farmer A (1997) Screening could seriously damage your health. BMJ 314: $533-534$

Stewart JB \& Chinnery PF (2015) The dynamics of mitochondrial DNA heteroplasmy: implications for human health and disease. Nat. Rev. Genet. 16: 530-542

Suomalainen A \& Battersby BJ (2018) Mitochondrial diseases: the contribution of organelle stress responses to pathology. Nat. Rev. Mol. Cell Biol. 19: 77-92

Tanaka M \& Ozawa T (1994) Strand asymmetry in human mitochondrial DNA mutations. Genomics 22: 327-335 Available at: http://dx.doi.org/10.1006/geno.1994.1391

Tang H \& Thomas PD (2016a) Tools for Predicting the Functional Impact of Nonsynonymous Genetic Variation. Genetics 203: 635-647

Tang H \& Thomas PD (2016b) PANTHER-PSEP: predicting disease-causing genetic variants using position-specific evolutionary preservation. Bioinformatics 32: 2230-2232

Turnbull DM, Taylor RW \& McFarland R (2018) Pickett S, Grady JP, Ng YS, Gorman GS, Schaefer AM, Wilson IJ, Cordell HJ. Annals of Clinical and Translational Neurology 5: 333-345

Vento JM \& Pappa B (2013) Genetic counseling in mitochondrial disease. Neurotherapeutics 10: $243-250$

Wallace DC (1994) Mitochondrial DNA mutations in diseases of energy metabolism. J. Bioenerg. Biomembr. 26: 241-250

Wei W \& Chinnery PF (2020) Inheritance of mitochondrial DNA in humans: implications for rare and common diseases. J. Intern. Med. Available at: http://dx.doi.org/10.1111/joim.13047

Wei W, Gomez-Duran A, Hudson G \& Chinnery PF (2017) Background sequence characteristics influence the occurrence and severity of disease-causing mtDNA mutations. PLoS Genet. 13: e1007126

Whiffin N, Minikel E, Walsh R, O’Donnell-Luria AH, Karczewski K, Ing AY, Barton PJR, Funke B, Cook SA, MacArthur D \& Ware JS (2017) Using high-resolution variant frequencies to empower clinical genome interpretation. Genet. Med. 19: 1151-1158

Williams GC (1957) Pleiotropy, Natural Selection, and the Evolution of Senescence. Evolution 11: $398-411$

Wolff JN, Ladoukakis ED, Enríquez JA \& Dowling DK (2014) Mitonuclear interactions: evolutionary consequences over multiple biological scales. Philos. Trans. R. Soc. Lond. 
B Biol. Sci. 369: 20130443

Ye K, Lu J, Ma F, Keinan A \& Gu Z (2014) Extensive pathogenicity of mitochondrial heteroplasmy in healthy human individuals. Proceedings of the National Academy of Sciences 111: 10654-10659 Available at: http://dx.doi.org/10.1073/pnas.1403521111

Zhang H, Burr SP \& Chinnery PF (2018) The mitochondrial DNA genetic bottleneck: inheritance and beyond. Essays Biochem. 62: 225-234

Zou Z \& Zhang J (2019) Amino acid exchangeabilities vary across the tree of life. Sci Adv 5: eaax3124

Zuckerkandl E \& Pauling L (1965) Evolutionary Divergence and Convergence in Proteins. In Evolving Genes and Proteins pp 97-166.

Zuk O, Schaffner SF, Samocha K, Do R, Hechter E, Kathiresan S, Daly MJ, Neale BM, Sunyaev SR \& Lander ES (2014) Searching for missing heritability: designing rare variant association studies. Proc. Natl. Acad. Sci. U. S. A. 111: E455-64 
A

\section{B}

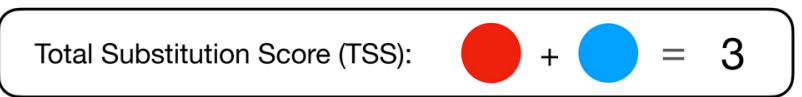

C

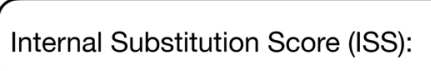

$$
=1
$$

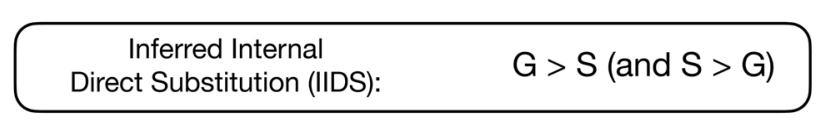

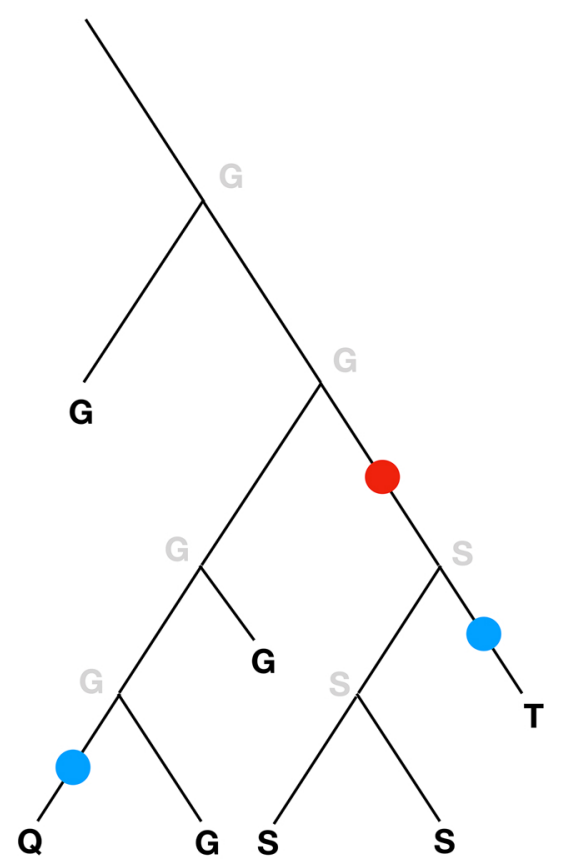

Figure 1: Calculation of Total Substitution Scores, Internal Substitution Scores, and Inferred Internal Direct Substitutions. Phylogenetic trees are produced for each macromolecule class (protein, rRNA, tRNA), then ancestral values were predicted for each node of the tree. Glycine is ancestral in this simplified example, with substitution to serine, threonine, and glutamine at internal or external branches. (A) Next, substitutions at an alignment position that occur at tree edges are summed to produce the total substitution score (TSS). (B) To minimize tabulation of artifactual changes related to alignment and sequencing errors, only substitutions at internal edges are summed to generate the internal substitution score (ISS). (C) Using the predicted node values, inferred internal direct substitutions (IIDSs) along any internal edge are determined, preferentially taking into account epistasis involving the site of interest and avoiding the effects of sequencing and alignment errors. 
bioRxiv preprint doi: https://doi.or ${ }^{400}[10.1101 / 2020.01 .10 .902239 ;$ this version posted October 10, 2020. The copyright holder for this preprint (which was not ceettified by peer review) is the author/funder. All rights reserved. No reuse allowed without permission.

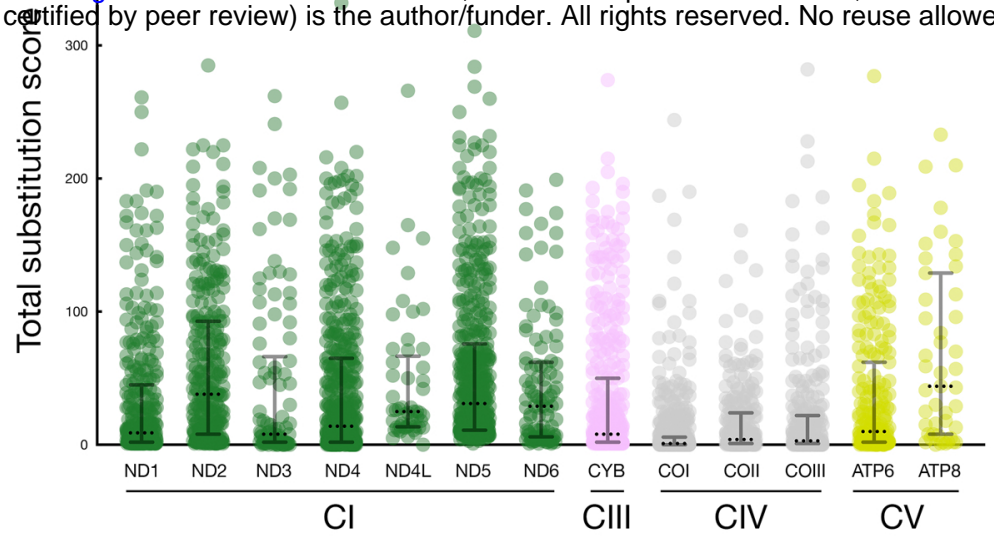

B

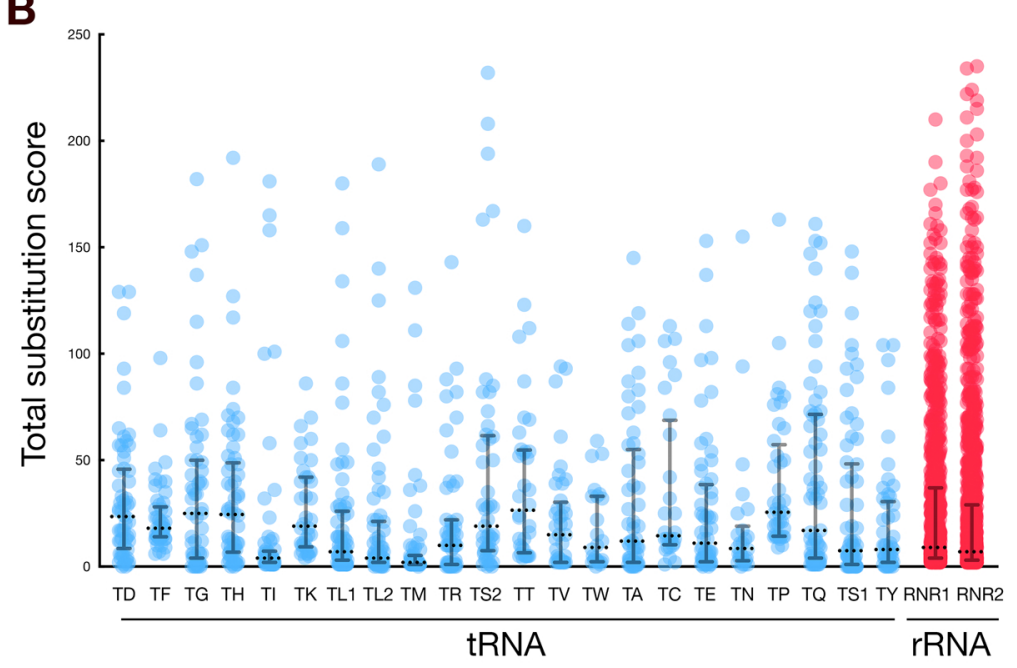

C

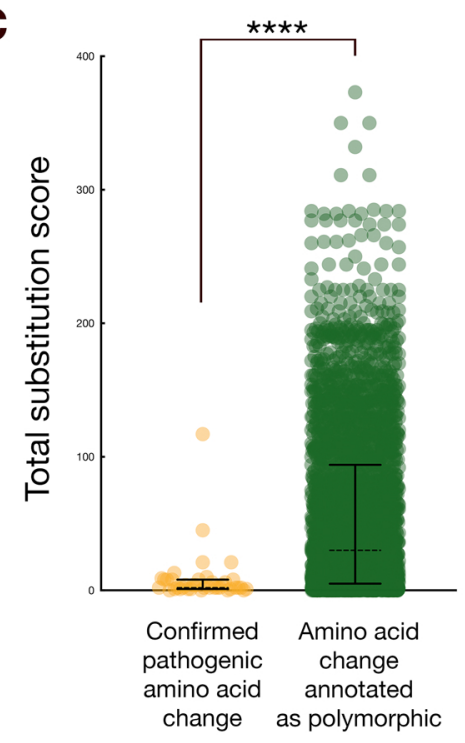

D

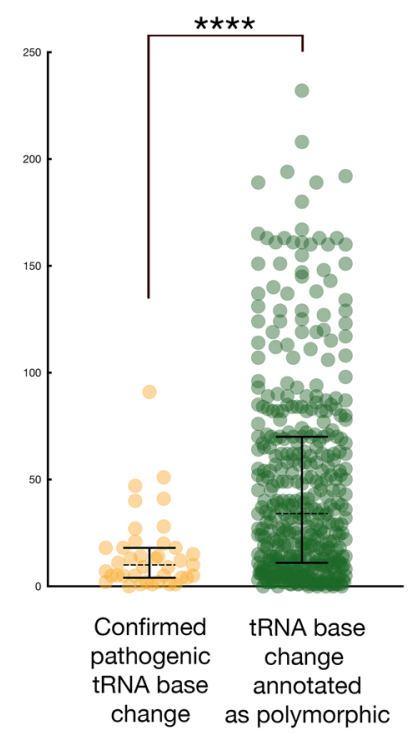

Figure 2: Most well-aligned positions encoded by the human mitochondrial genome are under selective constraint. (A) Nearly all amino acids in mammalian mtDNA-encoded proteins are under selection. The TSS analysis of alignment sites within mtDNA-encoded proteins, clustered by complex ( $89 \%$ of total amino acid positions, gapped $1.5 \%$ or less), are provided. (B) Nearly all ribonucleotides across mammalian mtDNA-encoded tRNAs and rRNAs are under selection. The TSS analysis of alignment sites within mtDNA-encoded tRNAs (75\% of total tRNA positions, gapped $1.5 \%$ or less) or rRNAs (77\% of total tRNA positions, gapped $1.5 \%$ or less) are provided. (C-D) Confirmed pathogenic mutations in protein and tRNAs are associated with low total substitution scores. TSSs for positions of variants annotated in the MITOMAP database as confirmed pathogenic substitutions or as polymorphisms are plotted for (C) protein positions analyzed using mammalian data and (D) tRNA positions analyzed using mammalian data. All analyzed positions are gapped at equal to or less than $1.5 \%{ }^{* \star * *}$, Kolmogorov-Smirnov test approximate P-value of $<0.0001$. Dotted horizontal lines, median, with solid horizontal lines providing the interquartile range. 
bioRxiv preprint doi: https://doi.org/10 1 $101 / 202 Q 01.10 .902239$; this version posted October 10,2020. The copyright holder for this preprint (which was not certified by peer review) is the author/funder. All rights reserved. No reusq allowed without permission.

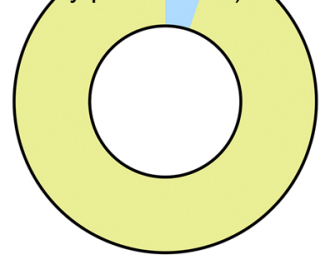

MITOMAP confirmed pathogenic protein change

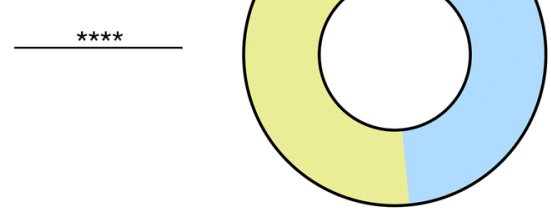

MITOMAP

protein 'polymorphism'

B

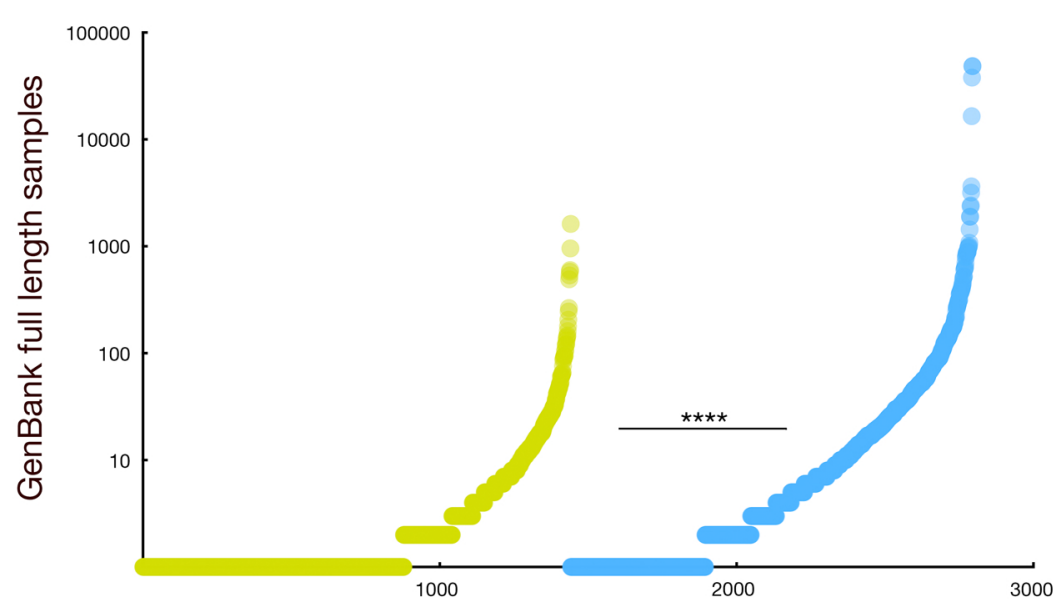

MITOMAP annotated protein 'polymorphism'

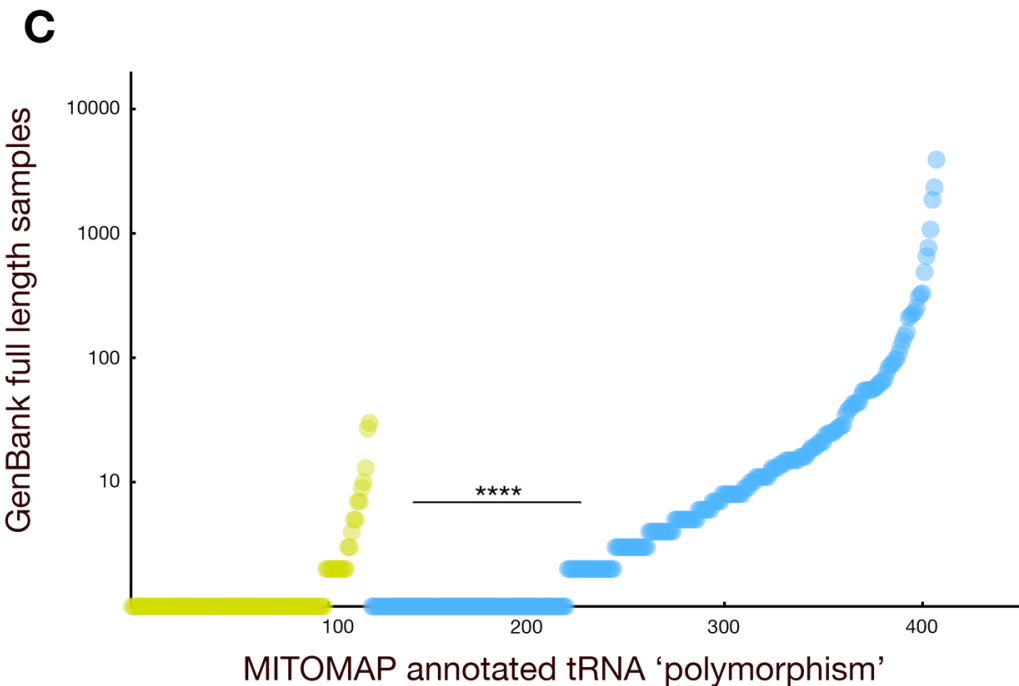

Figure 3: Analysis of inferred internal direct substitutions indicates that variants currently annotated as polymorphic may be deleterious. (A) Inferred internal direct substitutions within the mammalian phylogenetic tree correspond to a reduced likelihood of confirmed pathogenicity for mitochondrial protein coding variants. Confirmed pathogenic protein substitutions and substitutions currently annotated as polymorphisms, both retrieved from MITOMAP, were tested for the presence of an IIDS. All analyzed positions are gapped at equal to or less than 1.5\%. ${ }^{* \star *}$, Fisher's two-sided exact test P-value of $<0.0001$. (B) Population frequency distributions differ when amino acid substitutions from the human reference, which are currently annotated as polymorphic in MITOMAP, are classified based upon the presence or absence of an IIDS. All analyzed positions are gapped at equal to or less than $1.5 \%$. (C) as in (B), but for tRNA substitutions. ${ }^{* \star \star *}$, Kolmogorov-Smirnov test approximate P-value of $<0.0001$. 
A

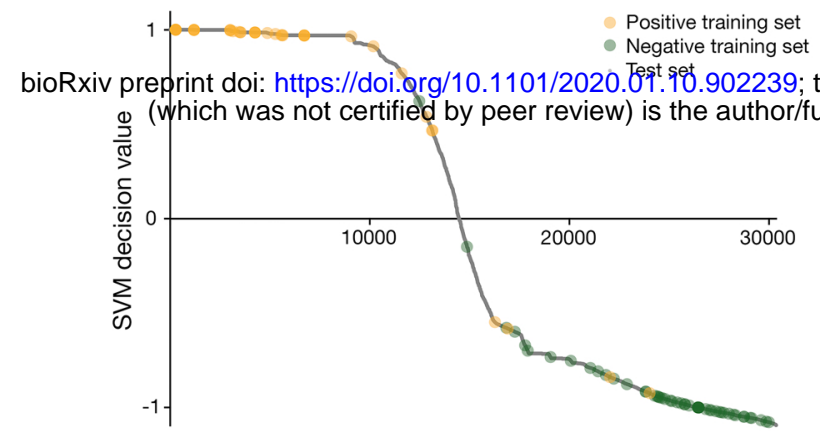

\section{B}

100 - Allele predicted pathogenic
8

sysion posted October 10, 2020. The copyright holder for this preprint

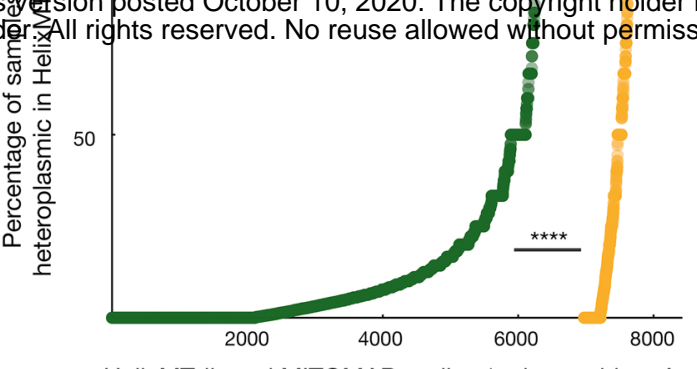

HelixMTdb and MITOMAP coding 'polymorphisms'

C

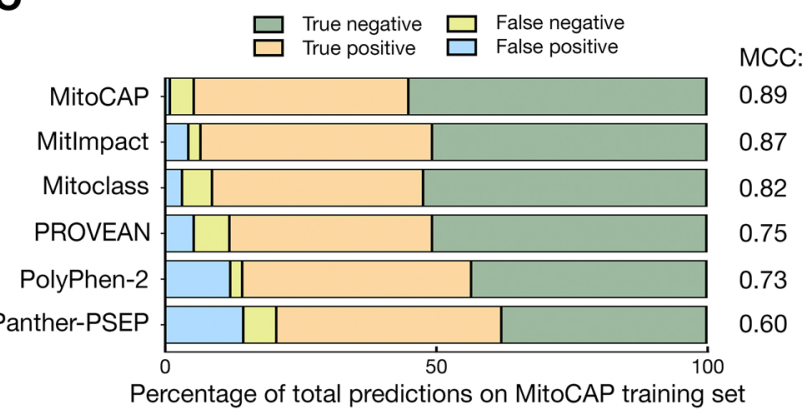

D

Percentage of total predictions on MitoCAP training set

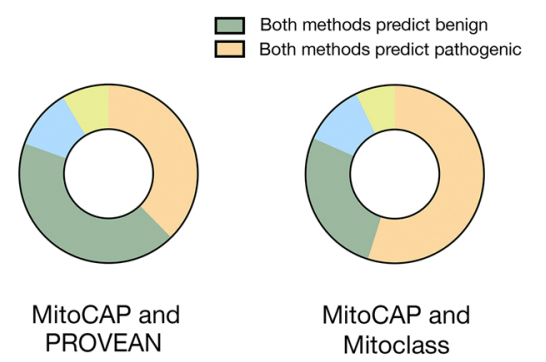

$\mathbf{E}$

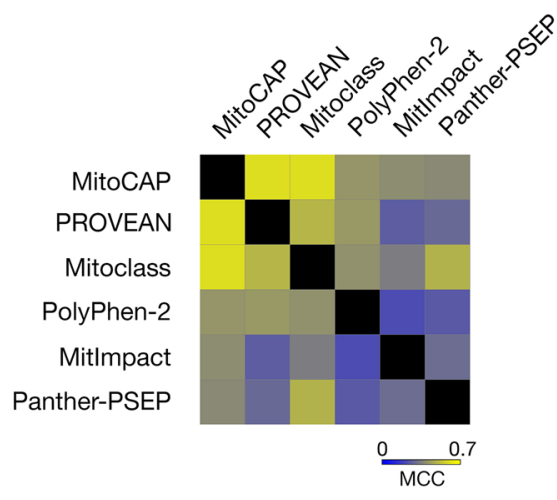

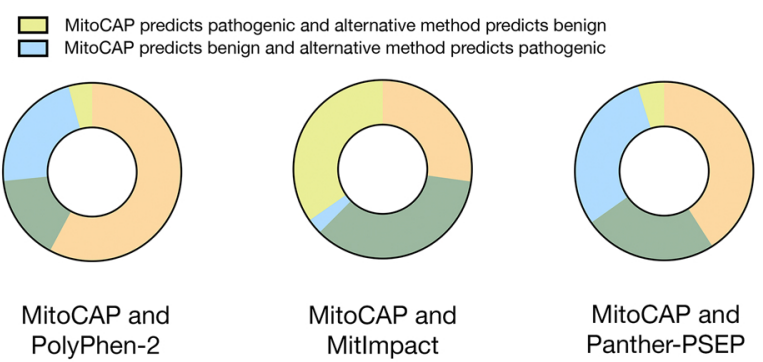

$\begin{array}{cc}\text { MitoCAP and } & \text { MitoCAP and } \\ \text { PolyPhen-2 } & \text { Mitlmpact }\end{array}$ Panther-PSEP

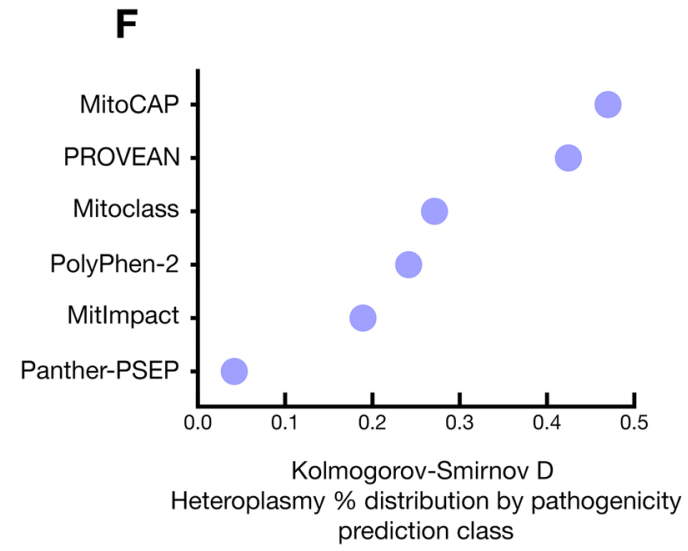

Figure 4: The MitoCAP classifier can successfully predict the pathogenicity of protein-coding mtDNA variants currently annotated as polymorphisms. (A) SVM-based prediction of pathogenicity for protein-coding variants effectively separates the positive and negative training sets, which are superimposed on the curve of variants and decision values. Variants are arrayed by decreasing SVM decision values on the x-axis. (B) MitoCAP classification of protein variants leads to distinct distributions of heteroplasmy propensity. The fractions of heteroplasmic HelixMTdb samples for a given variant are plotted in order of increasing value on the x-axis and separated by MitoCAP pathogenicity prediction. Plotted variants are annotated as 'polymorphic' in MITOMAP or are unannotated and do not belong to the positive or negative training sets. ${ }^{* \star \star \star}$, Kolmogorov-Smirnov test approximate P-value of $<0.0001$. (C) Comparison of protein substitution classifier behavior using the MitoCAP training set. The percentage of total predictions on the MitoCAP training set that are true negative, true positive, false negative, or false positive are reflected, as well as the calculated MCC. (D-E) MitoCAP predictions on substitutions in protein-coding genes are most similar to those of the PROVEAN classifier. (D) The fraction and characterization of instances where both approaches are in agreement or are discordant is represented. Both classifiers had to have made predictions in order for the comparison to enter the analysis, and all amino acid substitutions, including those of training sets, are included. (E) Comparison of prediction outcomes between each pair of methods, including all test and training set variants, was performed using MCC calculations and represented as a heat map. (F) MitoCAP classification of substitutions in mitochondrial proteins best differentiates heteroplasmy frequency distributions of unannotated variants. The Kolmogorov-Smirnov D statistic for each pair of classification-based distributions [(B) and Figure S5] is shown. The associated approximate P-values associated with each test are $<0.0001$, with the exception of Panther-PSEP $(P$ value $<0.05)$. 


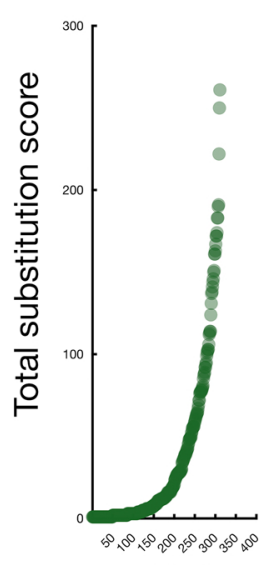

ND1

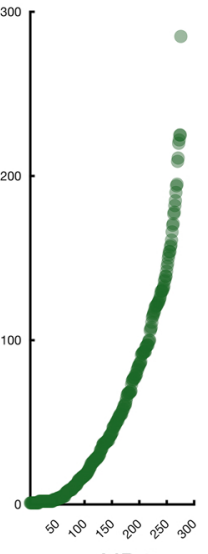

ND2

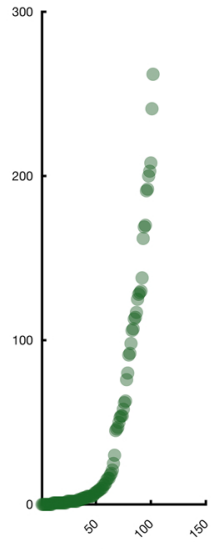

ND3

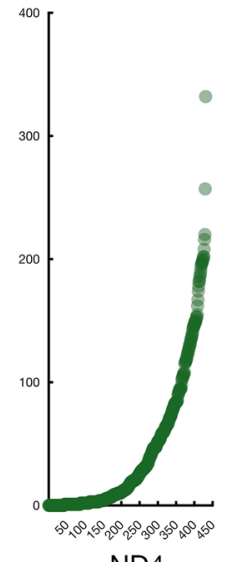

ND4

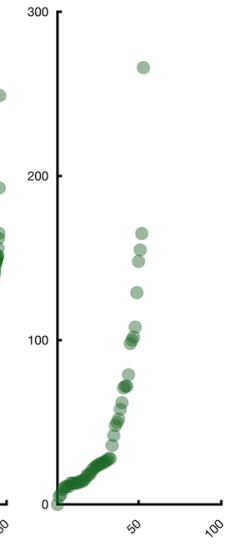

ND4L

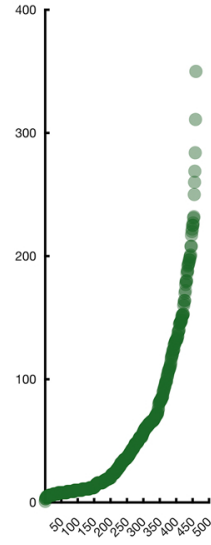

ND5

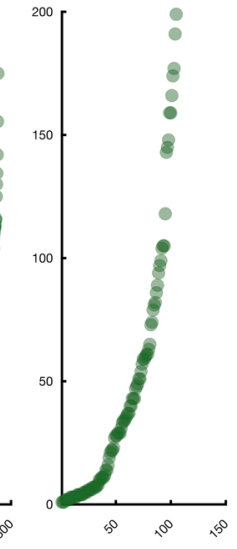

ND6

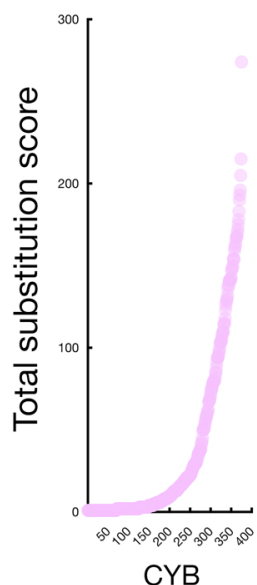

CYB

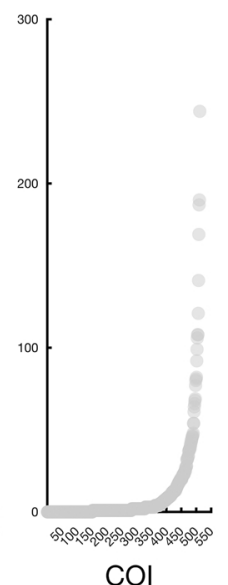

$\mathrm{COI}$

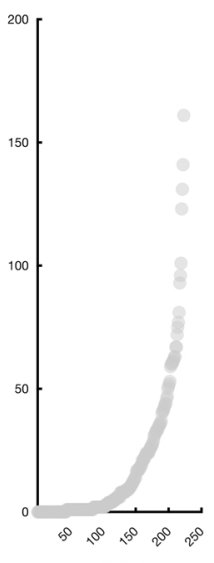

COII

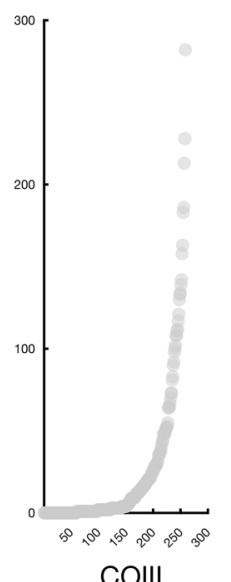

COIII

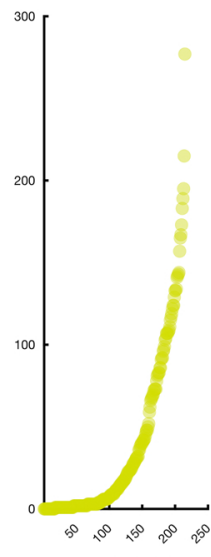

ATP6

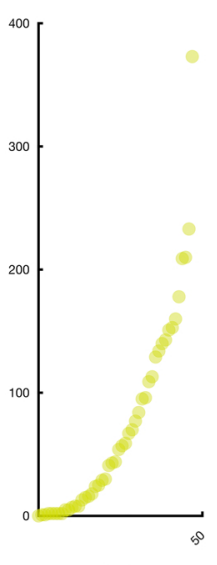

ATP8

Figure S1: Distributions associated with site TSS values demonstrate that the majority of mtDNA-encoded protein sites are under selection and that site saturation is minimal or absent. Site TSSs, also provided in Figure 2A, are plotted in increasing order for each protein. 


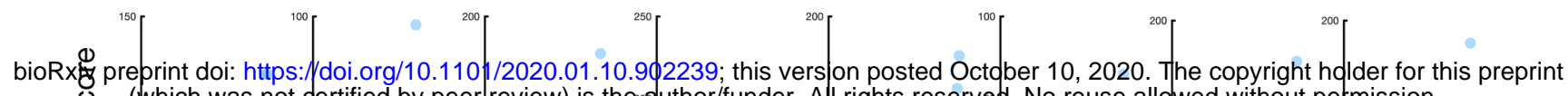

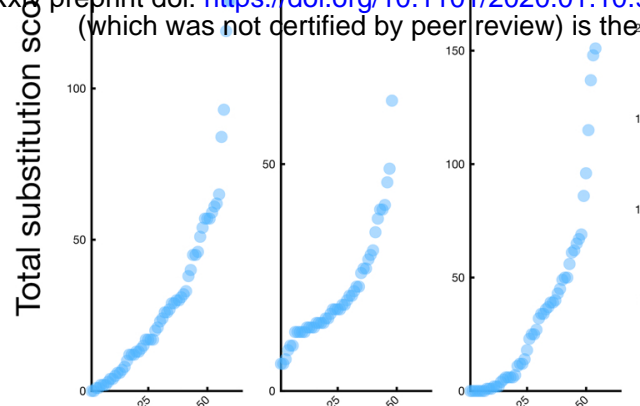

TD
TF

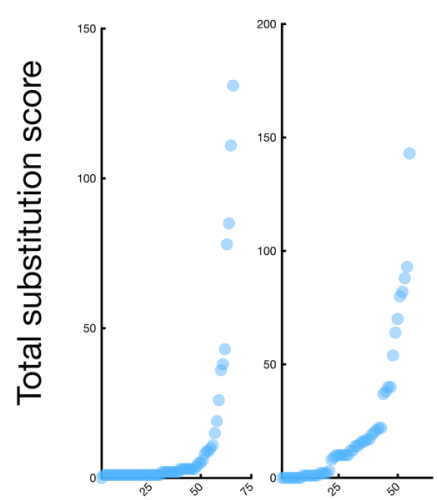

TM

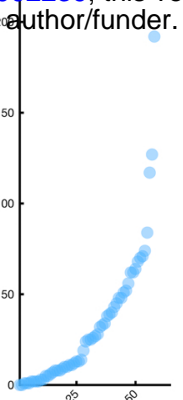

$\mathrm{TH}$

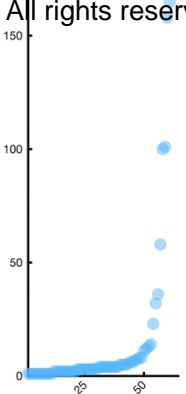

$\mathrm{TI}$

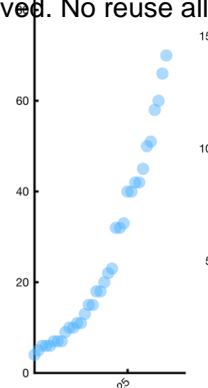

TK

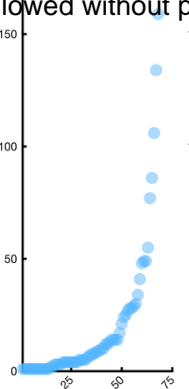

TL1

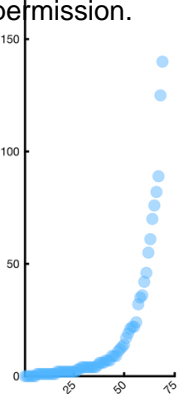

TL2

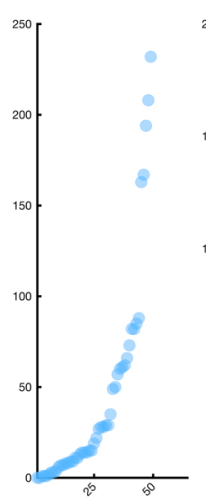

TS2

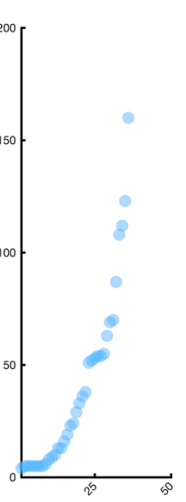

TT

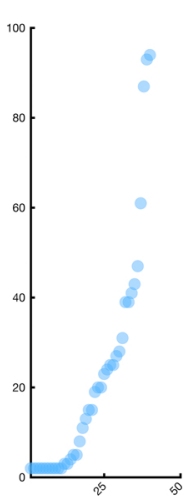

TV
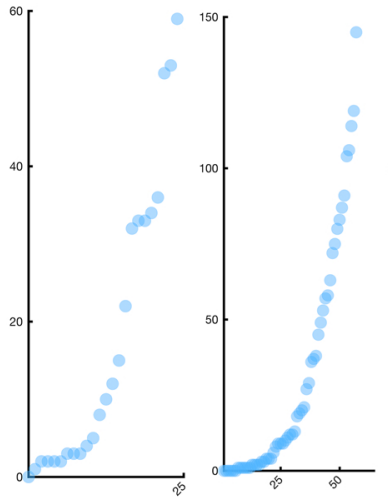

TA

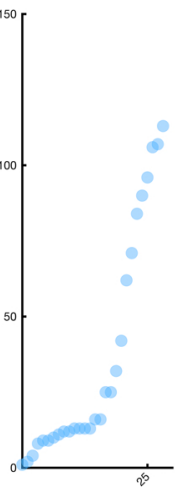

TC

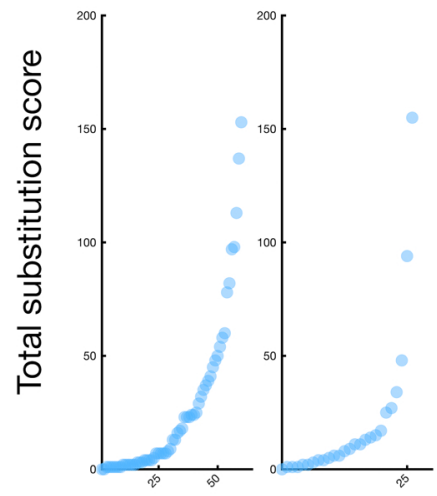

TE

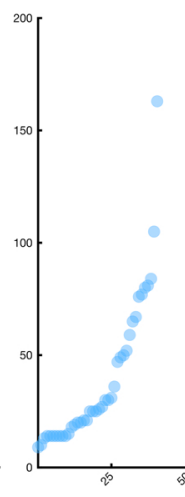

TP

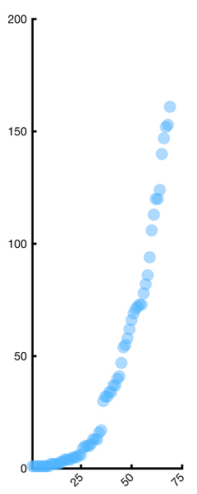

$T Q$

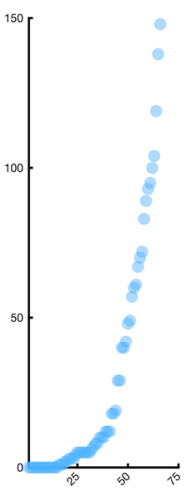

TS1

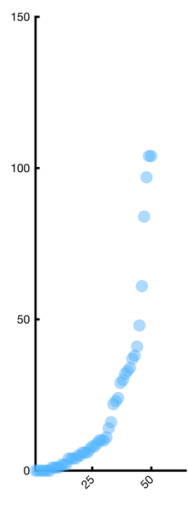

TY

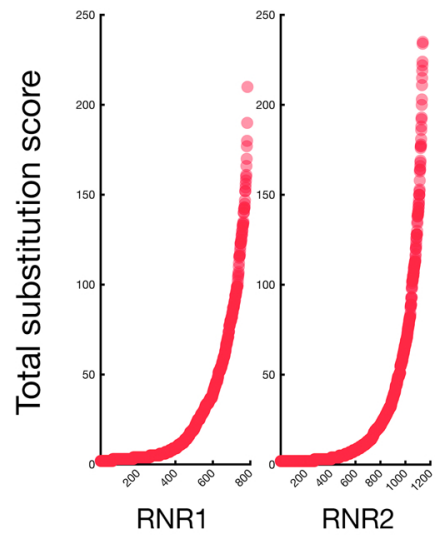

Figure S2: Distributions associated with site TSS values demonstrate that the majority of mtDNA-encoded RNA sites are under selection and that site saturation is minimal or absent. Plots are generated as in Figure S1, except tRNA and rRNA TSS distributions are shown. 
bioRxiv preprint doi: https://doi.org/10.1101/2020.01.10.902239; this version posted October 10, 2020. The copyright holder for this preprint $\mathbf{A}$ (which was not certified by peer review) is the author/fund $\mathbf{B}$. All rights reserved. No reuse allowed without permission. protein
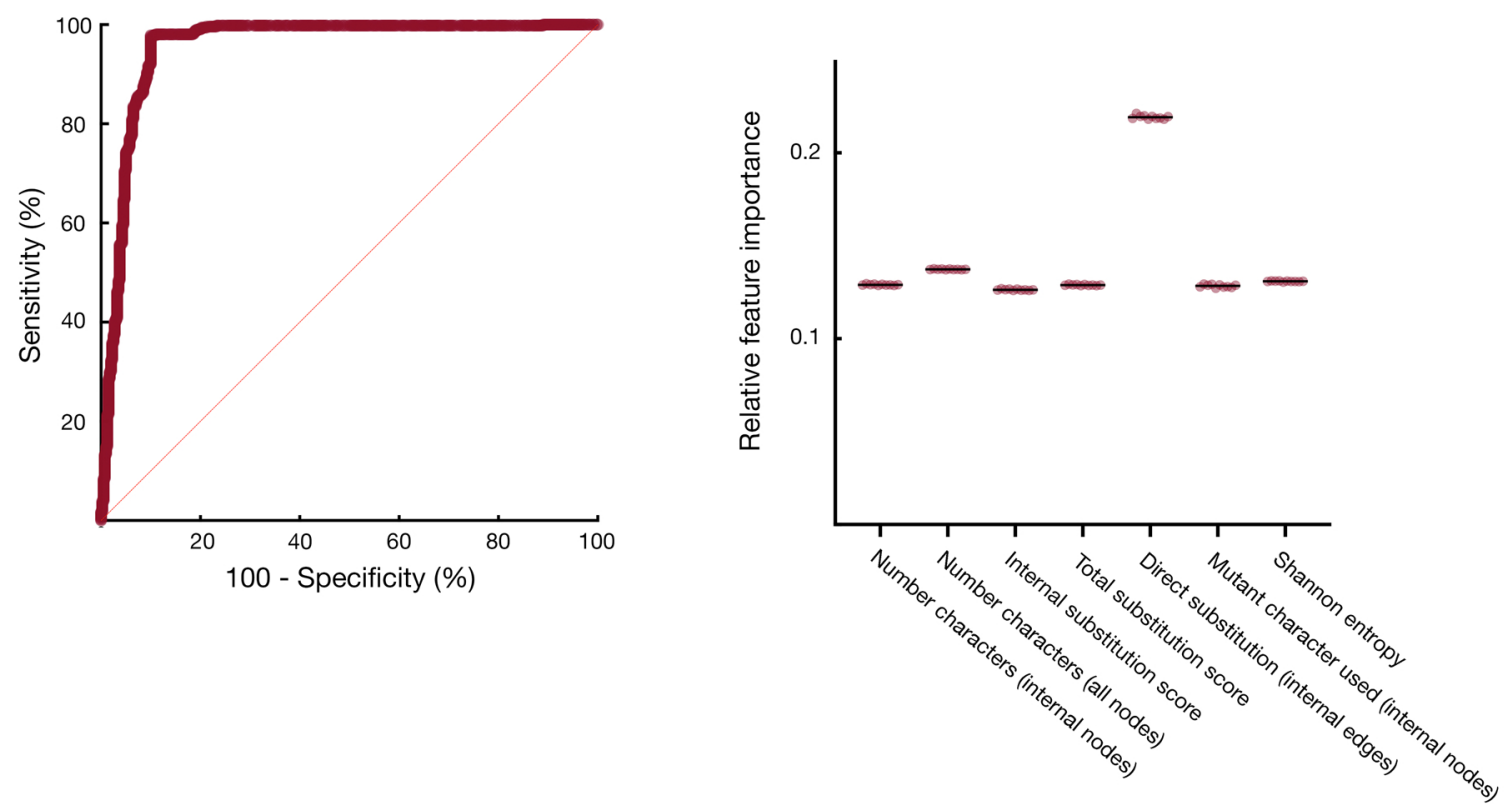

C

tRNA

D

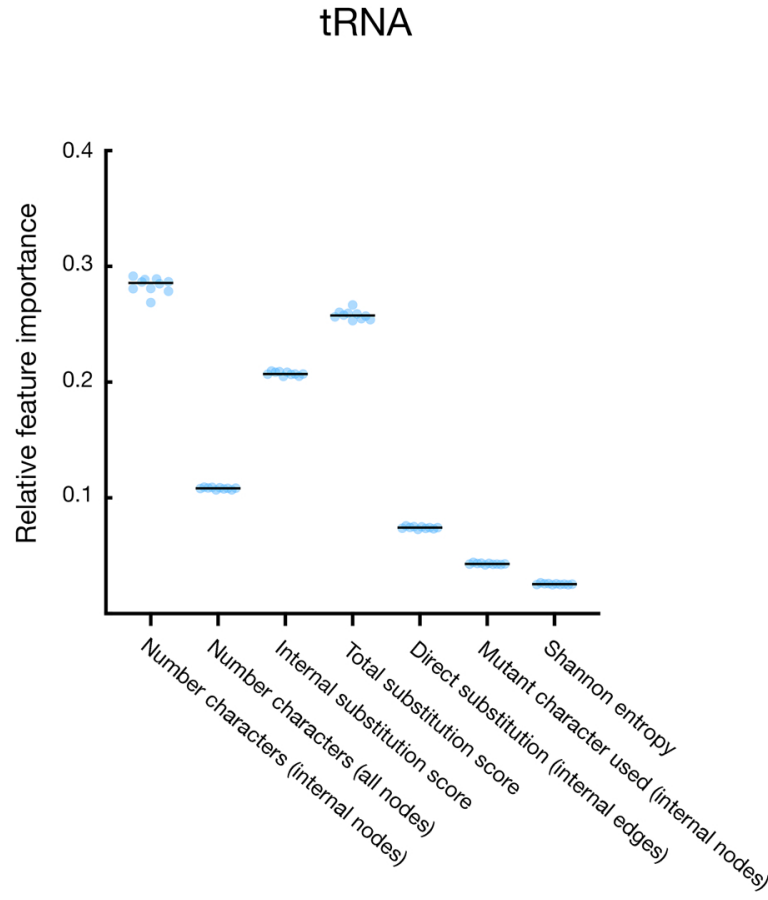

Figure S3: Feature importance and receiver operating characteristic curves for MitoCAP classification of protein and tRNA. (A) The ROC curve demonstrates very high sensitivity is achieved with no substantial loss in specificity for MitoCAP mammalian protein predictions. The true positive rate (sensitivity) of the predictive model is plotted against the false positive rate $(1$ - specificity) at various decision value thresholds. (B) Feature importance for prediction of protein-coding substitutions is calculated from the increase of the model's prediction error after varying the values for each feature. (C) as in (A), except the ROC curve associated with tRNA substitution predictions is shown. (D) as in (B), except feature importance for tRNA variant predictions is provided. 
bioRxiv preprint doi: https://doi.org/10.1101/2020.01.10.902239; this version posted October 10, 2020. The copyright holder for this preprint

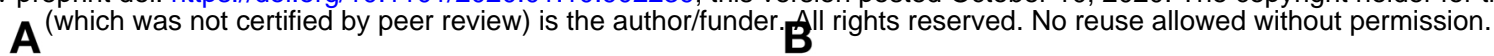

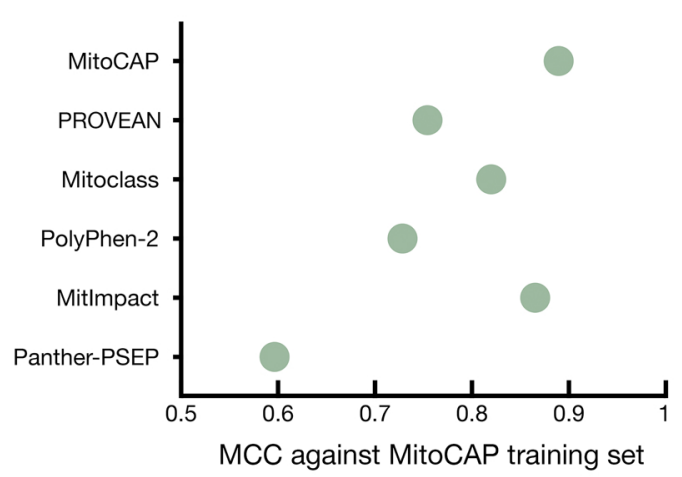

C

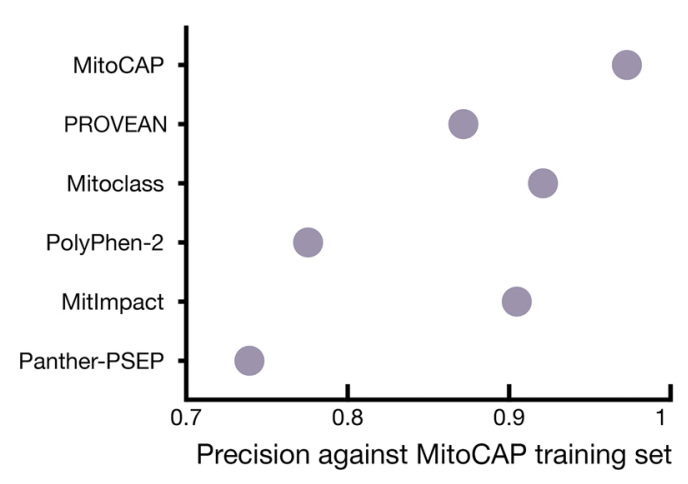

$\mathbf{E}$

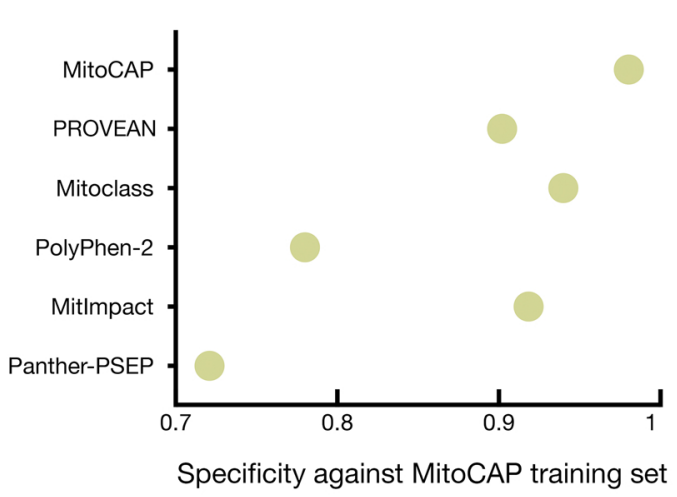

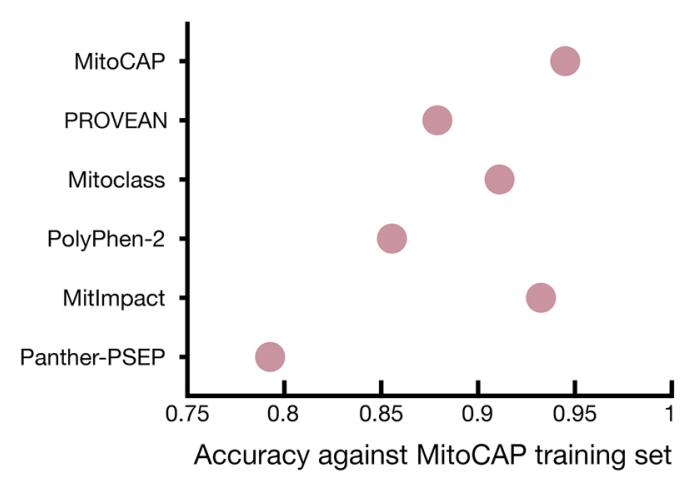

D

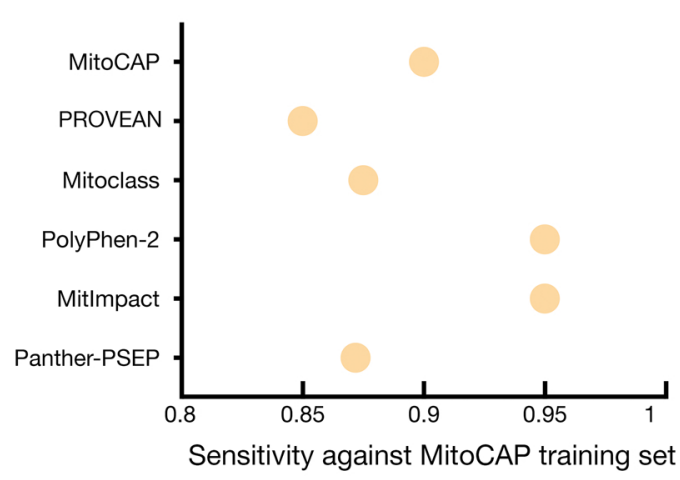

$\mathbf{F}$

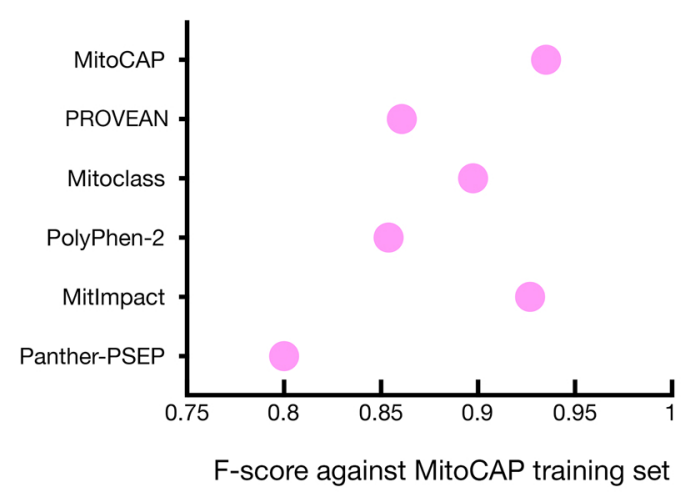

Figure S4: Metrics of classifier performance of MitoCAP and alternative methodologies using the MitoCAP training set for protein-coding substitutions. Plotted values obtained by the indicated classifiers include: (A) MCC, (B) accuracy, (C) precision, (D) sensitivity, (E) specificity, (F) F-score. 
bioRxiv preprint doi: https://doi.org/10.1101/2020.01.10.902239; this version posted October 10, 2020. The copyright holder for this preprint

A (which was not certified by peer review) is the author/fund $\mathbf{B}$. All rights reserved. No reuse allowed without permission. Provean

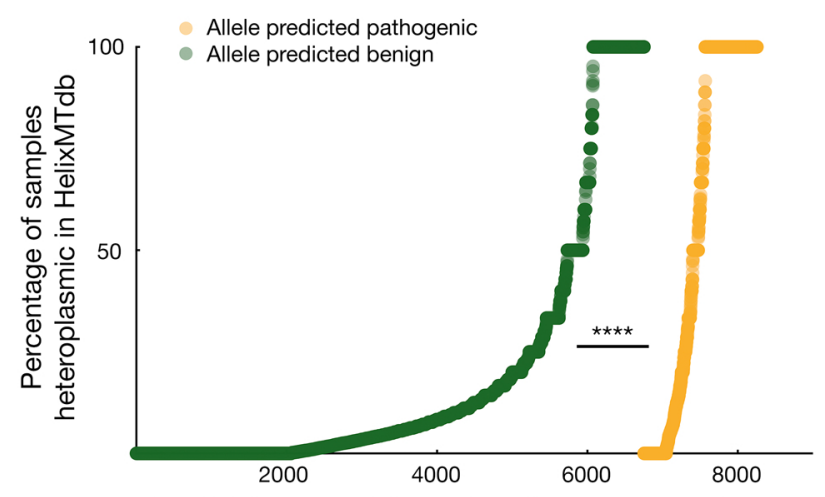

HelixMTdb and MITOMAP coding 'polymorphisms'

C

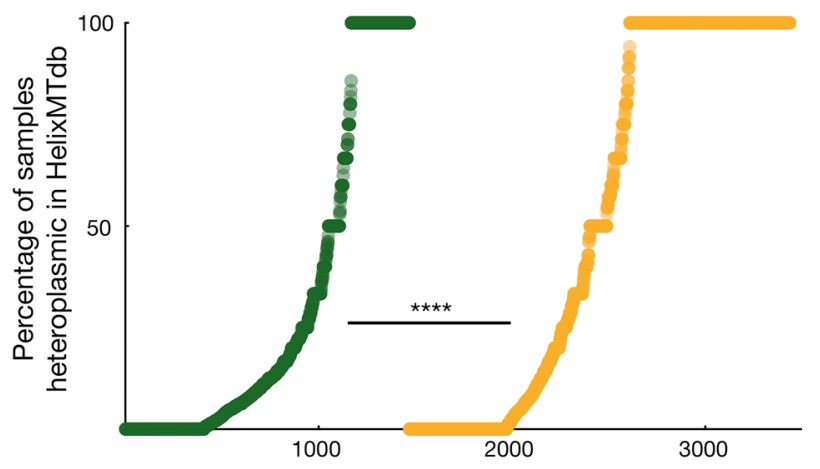

HelixMTdb and MITOMAP coding 'polymorphisms'

\section{$\mathbf{E}$}

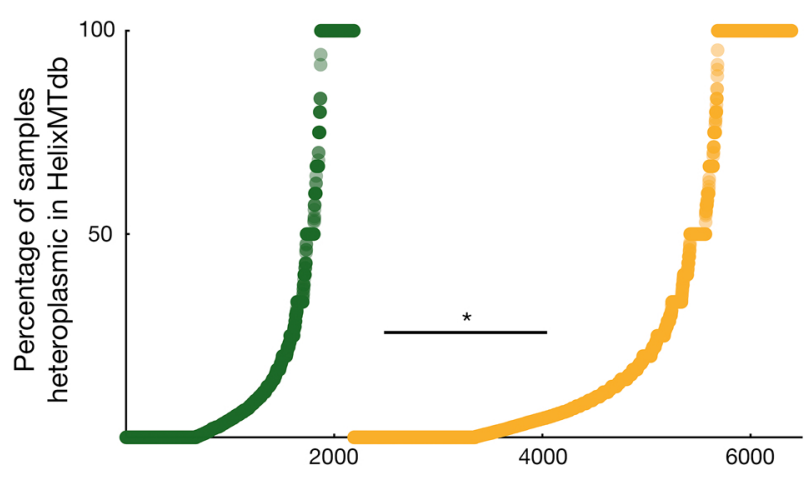

HelixMTdb and MITOMAP coding 'polymorphisms'

Panther-PSEP

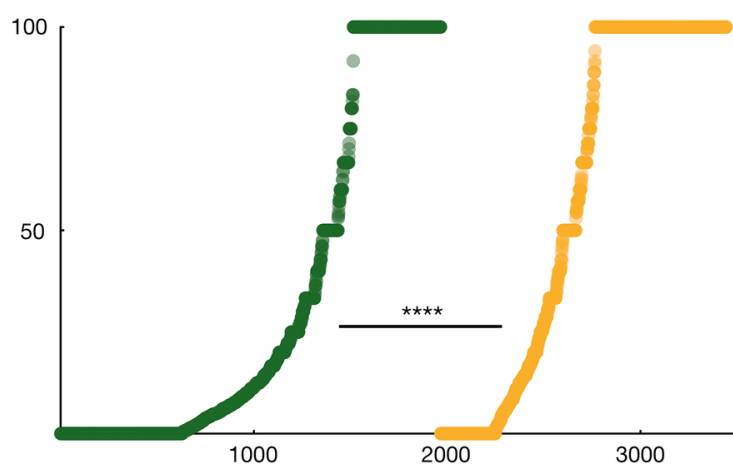

HelixMTdb and MITOMAP coding 'polymorphisms'

Ditlmpact

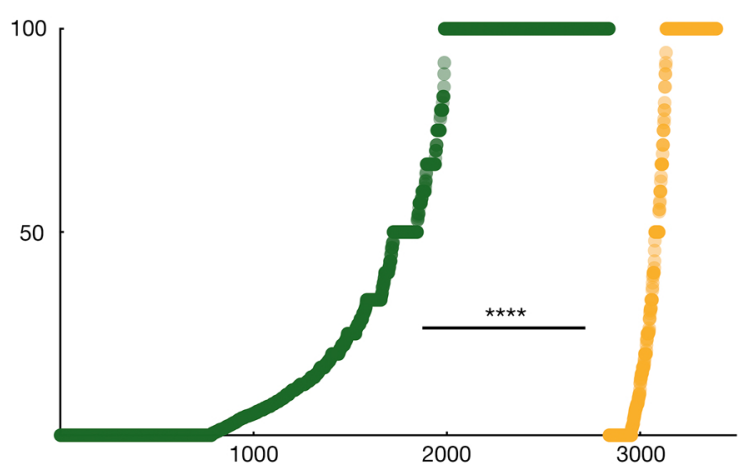

HelixMTdb and MITOMAP coding 'polymorphisms'

Figure S5: Separation of heteroplasmy frequency distributions for protein-coding substitutions by alternative classifiers. Distributions obtained using the indicated classifiers are plotted as in Figure 4B for (A) PROVEAN (B) Mitoclass (C) PolyPhen-2 (D) Mitlmpact (E) Panther-PSEP classifiers. 


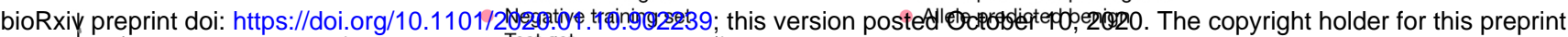
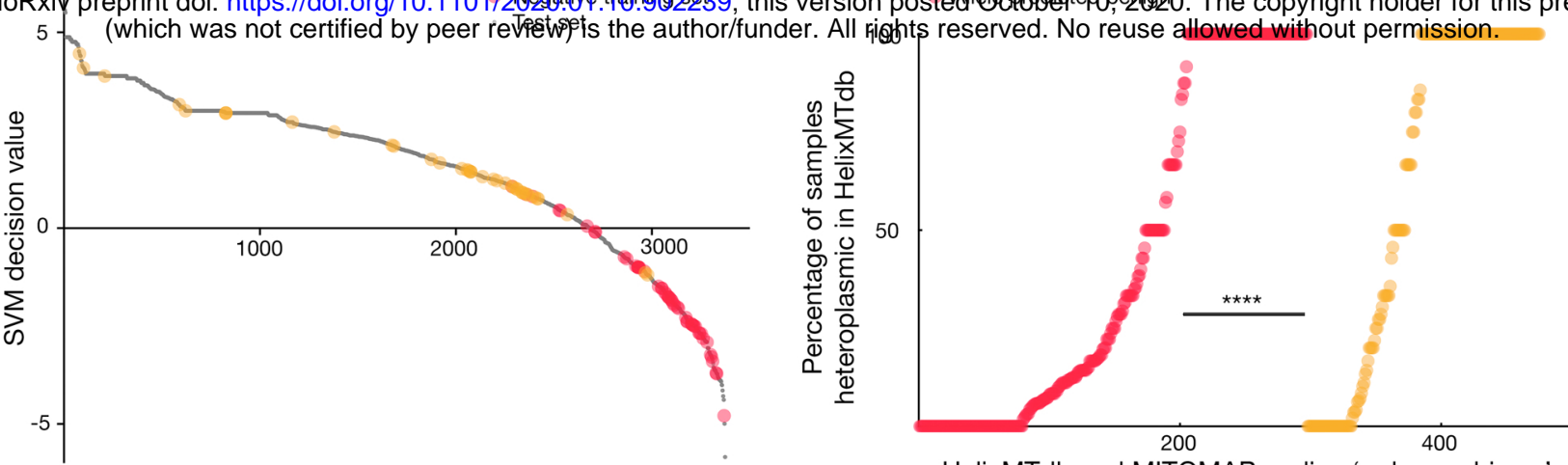

C

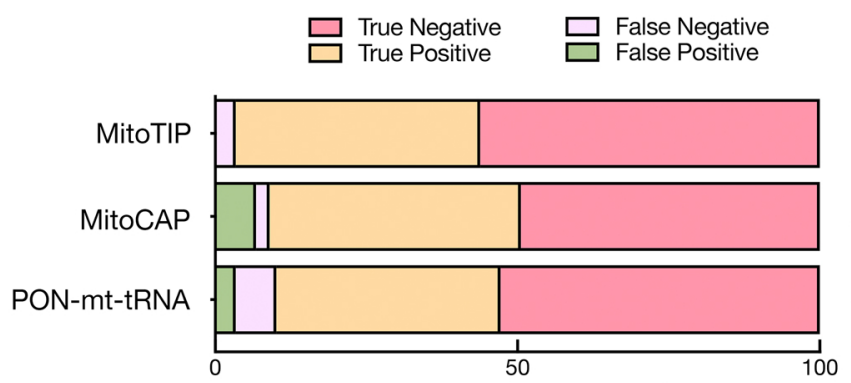

MCC:

0.93

0.82

Percentage of total predictions on MitoCAP training set

Both methods predict benign $\square$ MitoCAP predicts pathogenic and alternative method predicts benign

D

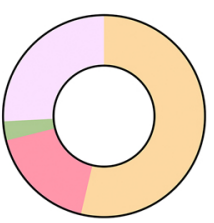

MitoCAP and

PON-mt-tRNA

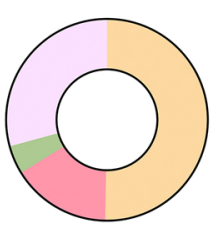

MitoCAP and MitoTIP
E

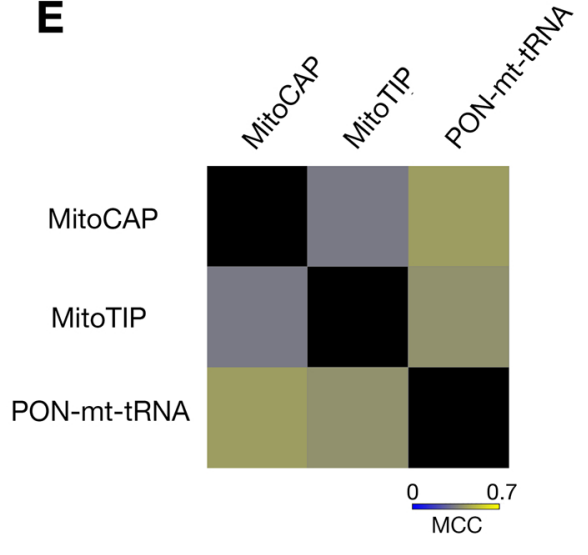

$\mathbf{F}$

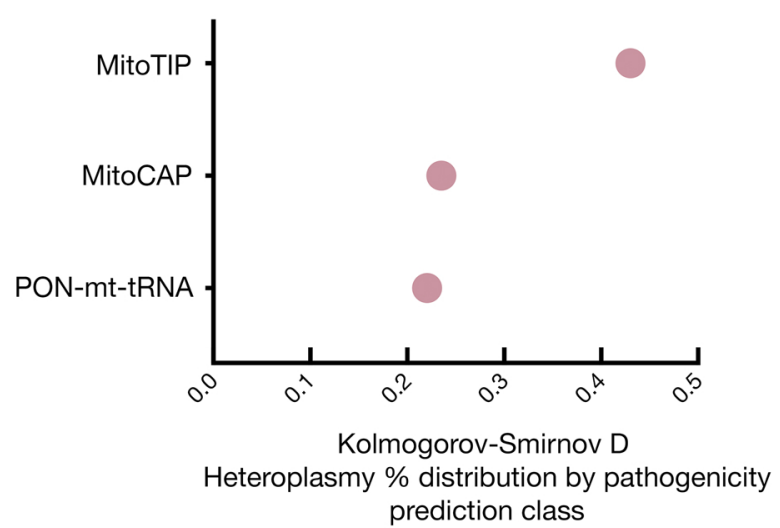

Figure S6: Prediction of mtDNA-encoded tRNA variants by the MitoCAP classifier. (A) as in Figure 4A, except that tRNA variants are plotted. (B) as in Figure 4B, except that tRNA variants are plotted. (C) as in Figure $4 \mathrm{C}$, except that the outcome of other methods is compared to that of MitoCAP on the MitoCAP tRNA training set. (D-E) as in Figure 4D and Figure 4E, except that comparisons are made between the outcome of tRNA prediction models. $(\mathrm{F})$ as in Figure 4F, except that distributions associated with heteroplasmy frequency and pathogenicity classification are made using tRNA substitution predictions. 
bioRxiv preprint doi: https://doi.org/10.1101/2020.01.10.902239; this version posted October 10, 2020. The copyright holder for this preprint (which was not certified by peer review) is the author/funder. All rights reserved. No reuse allowed without permission.

A

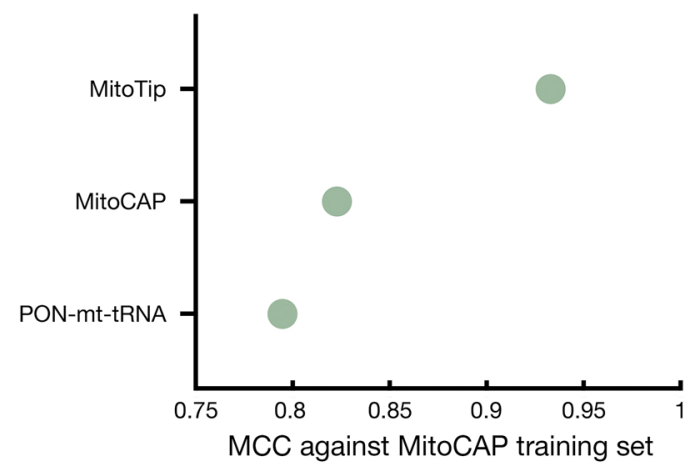

C

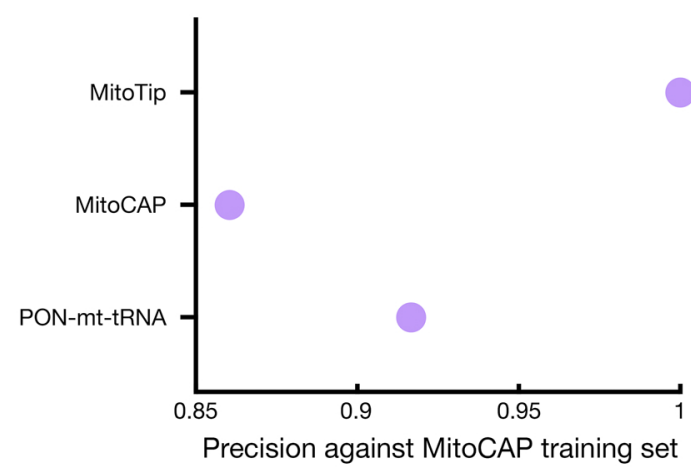

E

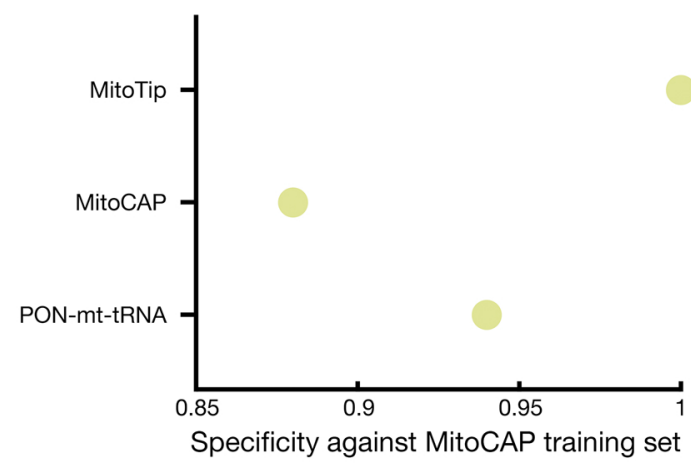

B

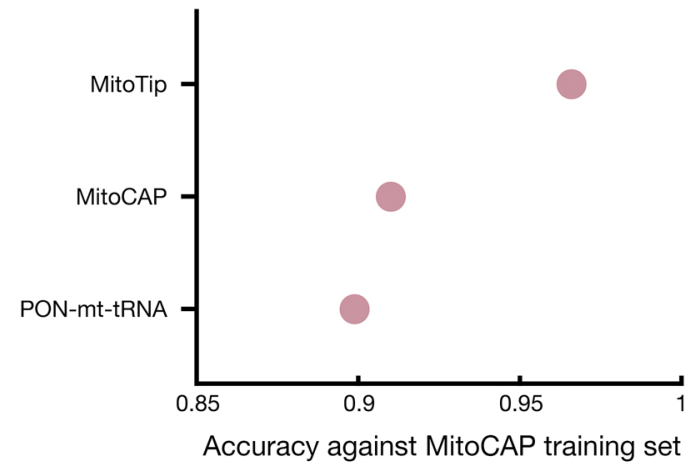

D

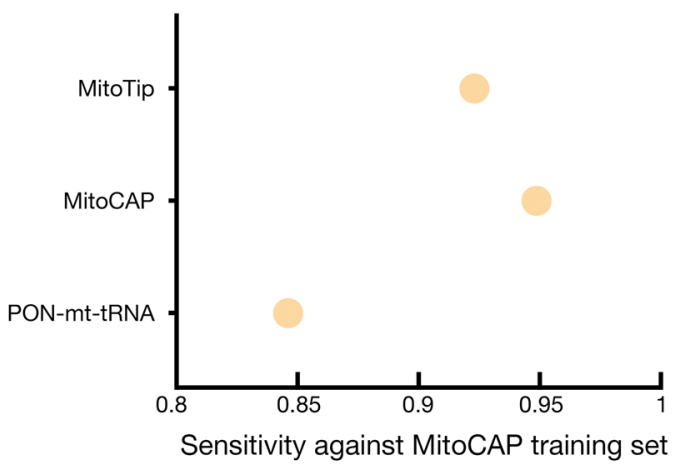

$\mathbf{F}$

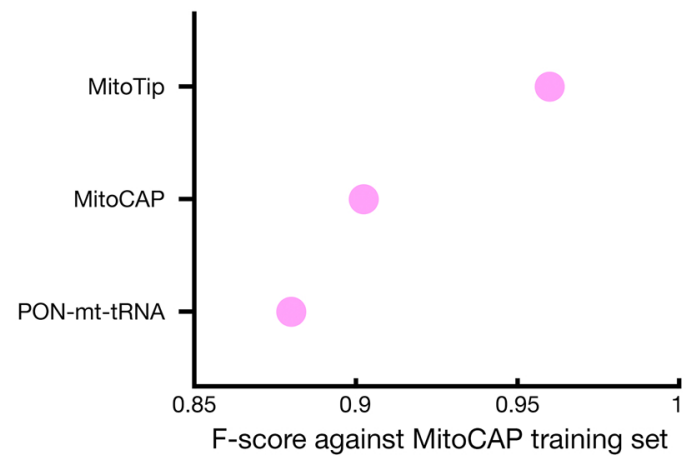

Figure S7: Metrics of classifier performance of MitoCAP and alternative methodologies on the MitoCAP training set for tRNA substitutions. Plotted values obtained by the indicated classifiers include: (A) MCC, (B) accuracy, (C) precision, (D) sensitivity, (E) specificity, (F) F-score. 
bioRxiv preprint doi: https://doi.org/10.1101/2020.01.10.902239; this version posted October 10, 2020. The copyright holder for this preprint (which was not certified by peer review) is the author/funder. All rights reserved. No reuse allowed without permission.

A

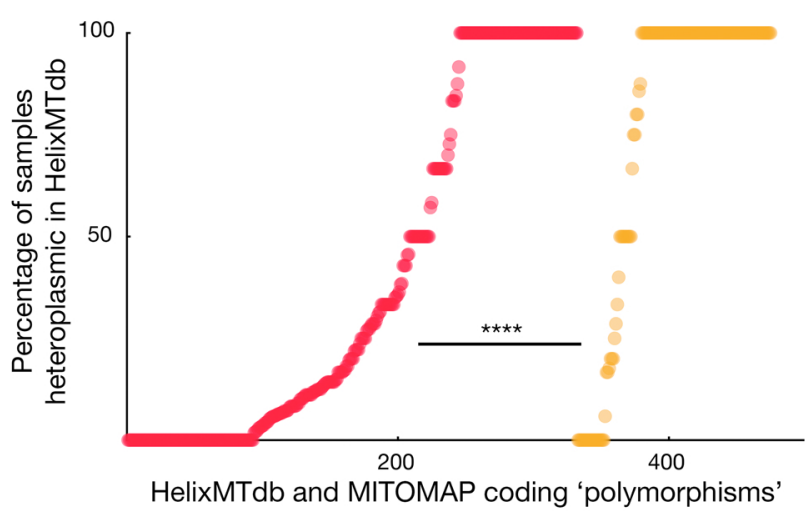

B

PON-mt-tRNA

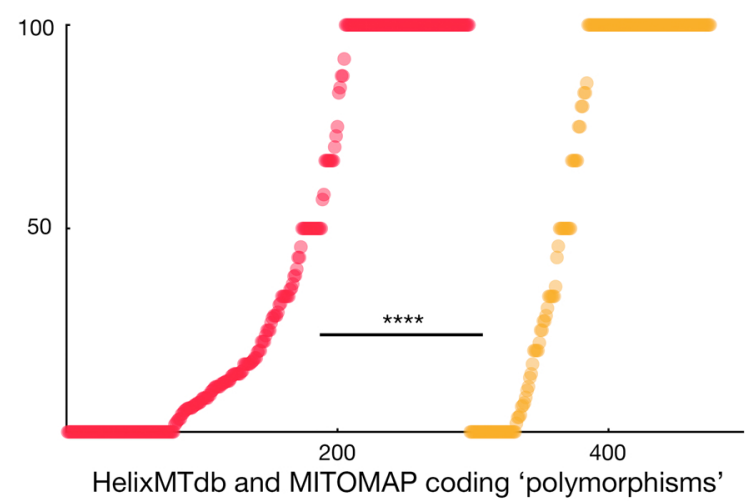

Figure S8: Separation of heteroplasmy frequency distributions for tRNA substitutions by alternative classifiers. Distributions obtained using the indicated classifiers are plotted as in Figure S6B for (A) MitoTIP and (B) PON-mt-tRNA classifiers. 
bioRxiv preprint doi: https://doi.org/10.1101/2020.01.10.902239; this version posted October 10, 2020. The copyright holder for this preprint (which was not certified by peer review) is the author/funder. All rights reserved. No reuse allowed without permission.
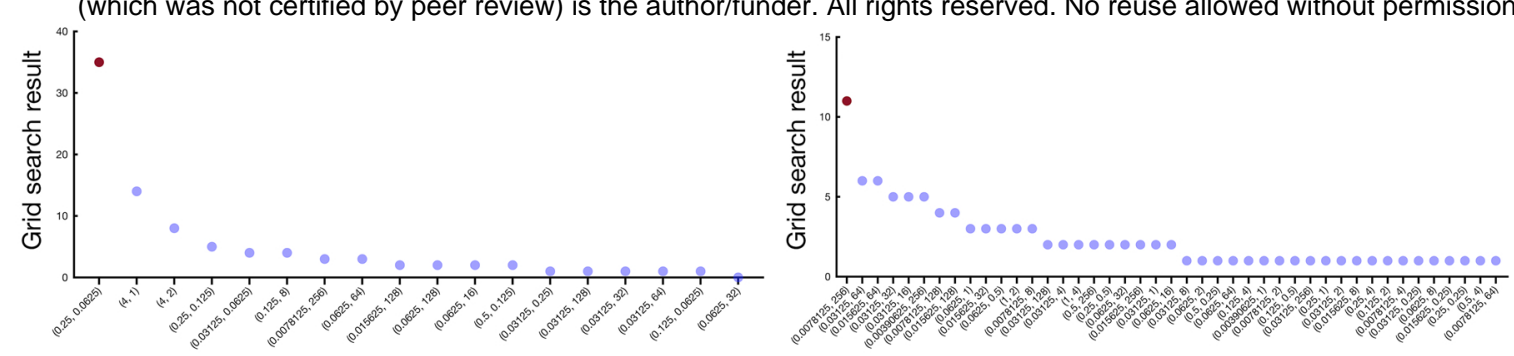

Optimized SVM parameters (gamma, cost) for protein prediction

Optimized SVM parameters (gamma, cost) for tRNA prediction
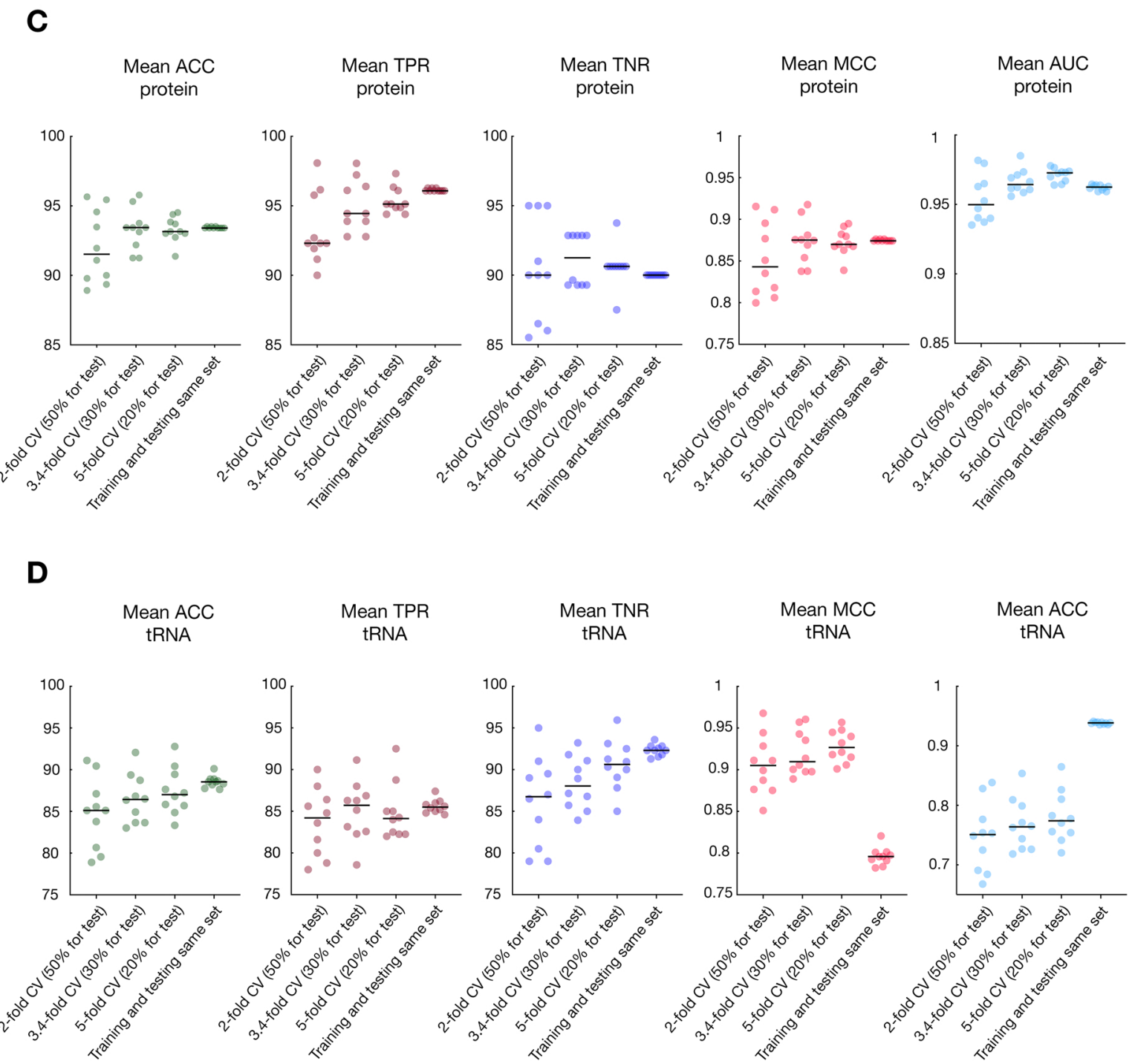

Figure S9: Optimization and validation of parameters used to develop the MitoCAP support vector machine classification model. (A) Optimal gamma and cost values for SVM model development were obtained before building the classifier. One hundred grid searches for optimal gamma and cost parameters were performed using the protein-coding substitution training dataset, and the values encountered the majority of the searches (red dot) were used during development of the classification model. (B) as for (A), but using the tRNA substitution training set. (C-D) Exploration of training set sufficiency demonstrates model stabilization using the full training sets. (C) k-fold cross-validation analyses were performed on the protein-coding variant training set to determine how robust classification might be to training set limitation. The mean values resulting from each instance of ten cross-validation tests with the indicated k-fold setting are plotted, and the bar indicates the median of these mean values. Mean accuracy (ACC), true positive rate (TPR), true negative rate (TNR), Matthews correlation coefficient (MCC), and area under the receiver operating characteristic curve (AUC) are plotted. (D) as in (C), except tRNA training sets were tested. 